\title{
Circular Scale of Time as a Guide for the Schrödinger Perturbation Process of a Quantum-Mechanical System
}

\author{
Stanis $\nmid$ aw Olszewski \\ Institute of Physical Chemistry, Polish Academy of Sciences, Warsaw, Poland \\ Email: olsz@ichf.edu.pl
}

How to cite this paper: Olszewski, S. (2019) Circular Scale of Time as a Guide for the Schrödinger Perturbation Process of a Quantum-Mechanical System. World Journal of Mechanics, 9, 113-145. https://doi.org/10.4236/wjm.2019.95009

Received: March 25, 2019

Accepted: May 13, 2019

Published: May 16, 2019

Copyright $\odot 2019$ by author(s) and Scientific Research Publishing Inc. This work is licensed under the Creative Commons Attribution International License (CC BY 4.0).

http://creativecommons.org/licenses/by/4.0/

\begin{abstract}
We point out that a suitable scale of time for the Schrödinger perturbation process is a closed line having rather a circular and not a conventional straight-linear character. A circular nature of the scale concerns especially the time associated with a particular order $N$ of the perturbation energy which provides us with a full number of the perturbation terms predicted by Huby and Tong. On the other hand, a change of the order $N$-connected with an increased number of the special time points considered on the scale-requires a progressive character of time. A classification of the perturbation terms is done with the aid of the time-point contractions present on a scale characteristic for each $N$. This selection of terms can be simplified by a partition procedure of the integer numbers representing $N-1$. The detailed calculations are performed for the perturbation energy of orders $N=7$ and $N=8$.
\end{abstract}

\section{Keywords}

Quantum Mechanics, Schrödinger's Perturbation Process, Accuracy of a Circular Scale of Time in the Perturbation Calculations

\section{Introduction}

The scale of time, which is well known in everyday life and in science, too, is a product of a long experience. As far as we can distinguish the later events from the earlier ones, we organize the idea of time as a parameter which allows us to get an insight into the degree of the past, or future, connected with our observations.

In effect a tool to classify the events, and the time distances between them, is established. Conventionally this is done with the aid of an infinite scale extended between an infinite past-say representing the negative coordinates-and a sim- 
ilar scale-say having the positive coordinates-representing the future:

$$
-\infty<t<\infty \text {. }
$$

The distances between the time points on the scale can be measured with a smaller or larger accuracy. These distances provide us with separations between different time points.

In practice the Schrödinger's quantum mechanics-developed in course of 1920's [1] [2] [3] [4] - has not much to do with the intervals of time. Its main idea was rather to distinguish between the stationary states of the chosen pieces of matter. Such pieces are described with the aid of the stationary eigenenergies and eigenfunctions, both kinds of parameters being independent of time. Concretely the classical Hamiltonian function of a chosen object is transformed into its operator form, and the integration of the classical Hamilton equations is replaced by a study of a differential eigenequation of the form

$$
\hat{H} \psi=E \psi .
$$

Here $\hat{H}$ is the Hamiltonian operator represented by a sum of the kinetic and potential operators

$$
\hat{H}=\hat{E}_{\text {kin }}+\hat{E}_{\text {pot }},
$$

so-for a single particle system-

$$
\begin{gathered}
\hat{E}_{\text {kin }}=\frac{1}{2 m}\left(\hat{p}_{x}^{2}+\hat{p}_{y}^{2}+\hat{p}_{z}^{2}\right), \\
\hat{E}_{\mathrm{pot}}=\hat{V}(\boldsymbol{r})=V(\boldsymbol{r}),
\end{gathered}
$$

$\psi$ is the eigenfunction called the wave function of an object, say a particle submitted to an external field having the potential $V$, symbol $r$ is the position vector, $E$ is the energy eigenvalue.

Because of

$$
\hat{p}_{x}=-i \hbar \frac{\partial}{\partial x}, \quad \hat{p}_{y}=-i \hbar \frac{\partial}{\partial y}, \quad \hat{p}_{z}=-i \hbar \frac{\partial}{\partial z},
$$

the momentum operator in (4) is of a differential character, whereas (5) represented by a function of the particle (object) position $\boldsymbol{r}$, is of a multiplicative nature.

The problem is that even in relatively simple physical cases the eigenequation (2) is difficult to solve. By solution we understand a set of the eigenenergies

$$
E=E_{1}, E_{2}, E_{3}, \cdots
$$

and eigenfunctions

$$
\psi=\psi_{1}, \psi_{2}, \psi_{3}, \cdots
$$

which satisfy (2). Only in very few physical cases equation (2) can provide us with simple solutions (7) and (8). The (7) are considered to be real energies of the system's quantum states, the (8) are the wave functions suitable in calculating other physical observables than energy.

In general the case when all eigenvalues on the right of (7) are different is classified as a non-degenerate problem, an opposite case is called to be a dege- 
nerate one.

Schrödinger was certainly aware about the difficulties connected with the solution of his Equation (2); see [3]. His proposal became to calculate the solutions of a rather complicated (2) with the aid of solutions of a less complicated equation

$$
\hat{H}^{(0)} \psi^{(0)}=E^{(0)} \psi^{(0)}
$$

having the potential $V^{0}(\boldsymbol{r})$ more simple than $V(\boldsymbol{r})$ in (2). The potentials difference

$$
\hat{V}^{\text {per }}=V^{\text {per }}=V(\boldsymbol{r})-V^{(0)}(\boldsymbol{r})
$$

is called the perturbation potential, or simply a perturbation. In order to obtain possibly accurate results Schrödinger developed a formalism in which the solutions of (2) can be expressed with the aid of solutions of (2a). In this process-beyond of the solutions of $(2 \mathrm{a})$ - the matrix elements of the kind

$$
\left\langle\psi_{\alpha}^{(0)}\left|V^{\text {per }}\right| \psi_{\beta}^{(0)}\right\rangle
$$

are also involved.

A more easy treatment of the perturbation does concern the calculation of the energies of Equation (2) with the aid of solutions of Equation (2a) obtained in case of a non-degenerate case. Nevertheless an accurate calculation of these energies requires a complicated superposition of the solutions of $(2 a)$, as well as calculation of the matrix elements in (10). In principle these calculations were performed with no reference to the parameter of time; see Sec. 2.

The aim of the present paper is to point out that an introduction of the time scale-which has, however, a nature different than the well-known scale characterized by the formula (1) - provides us with a rather spectacular simplification of the original Schrödinger's perturbation scheme.

\section{Outline of the Time-Independent Perturbation Theory of a Non-Degenerate Quantum State}

A characteristic point is that Schrödinger obtained the solution of his perturbed equation without any reference to time [3]. An outline of a more modern time-independent perturbation theory is given, for example, in [5]. In the case of a non-degenerate quantum system let the unperturbed eigenequation

$$
\hat{H}^{(0)} \psi_{n}^{(0)}=E_{n}^{(0)} \psi_{n}^{(0)}
$$

be considered as solved. In principle we have an infinite set of the quantum numbers $n$ for wnich the eigenequation (11) does hold. The number of the eigenfunctions and eigenenergies of the perturbed eigenequation

$$
\hat{H}(\lambda) \psi=\left(\hat{H}_{0}+\lambda V^{\text {per }}\right) \psi=E \psi
$$

let be also infinite.

For $\lambda=0$ we obtain the unperturbed problem equivalent to (11), whereas for $\lambda=1$ we have a full perturbation problem. In principle we assume as valid 
the following series expansions

$$
\psi_{n}(\lambda)=\psi_{n}^{(0)}+\lambda \psi_{n}^{(1)}+\lambda^{2} \psi_{n}^{(2)}+\cdots
$$

and

$$
E_{n}(\lambda)=E_{n}^{(0)}+\lambda \Delta E_{n}^{(1)}+\lambda^{2} \Delta E_{n}^{(2)}+\cdots
$$

and look for the solution of (12) in terms of the functions

$$
\psi_{n}^{(0)}, \psi_{n}^{(1)}, \psi_{n}^{(2)}, \cdots
$$

and numbers

$$
E_{n}^{(0)}, \Delta E_{n}^{(1)}, \Delta E_{n}^{(2)}, \cdots
$$

which make (12) valid for any $\lambda$ from the interval

$$
0<\lambda<1 \text {. }
$$

The function combined on the right of (13), viz.

$$
\psi_{n}=\psi_{n}^{(0)}+\psi_{n}^{(1)}+\psi_{n}^{(2)}+\cdots+\psi_{n}^{(N)}
$$

is called the perturbed wave function of state $n$ presented with the accuracy to the perturbation order $N$, whereas the numbers entering on the right of (14), viz.

$$
E_{n}=E_{n}^{(0)}+\Delta E_{n}^{(1)}+\Delta E_{n}^{(2)}+\cdots+\Delta E_{n}^{(N)}
$$

give the perturbed energy of state $n$ also with the accuracy of the perturbation order $N$.

By assuming the convergence of the series in (13) and (14), an increase the order number $N$ applied in the sequence

$$
1,2,3, \cdots, N
$$

improves the accuracy of solutions presented in (13) and (14).

Physically as a more easy accessible and more interesting parameter, is considered the perturbed energy (14). Huby and Tong presented the number of the kinds of terms necessary to obtain the successive components

$$
\Delta E^{(1)}, \Delta E^{(2)}, \Delta E^{(3)}, \cdots \Delta E^{(N)},
$$

entering the Schrödinger series for the energy perturbation of any non-degenerate state $n$; see [6] [7]. This number is expressed as a function of $N$ by the formula

$$
S_{N}=\frac{(2 N-2) !}{N !(N-1) !} .
$$

For low $N$ the numbers $S_{N}$ are also rather small, for example

$$
S_{1}=1, S_{2}=1, S_{3}=2, S_{4}=5, S_{5}=14, S_{6}=42, \cdots
$$

It should be noted that the kinds of the perturbation terms entering the set of $S_{N}$ do not depend explicitly on state $n$, but they depend solely on $N$. Any kind of terms is, in its turn, a combination of the matrix elements of the perturbation potential with the unperturbed wave functions $\psi_{\alpha}^{(0)}$ and $\psi_{\beta}^{(0)}$, given in (10).

Another dependence of the terms is due to the differences of the unperturbed energy $E_{n}^{(0)}$ of state $n$ submitted to perturbation and similar unperturbed ener- 


$$
\begin{aligned}
\text { gies } E_{\alpha}^{(0)}, E_{\beta}^{(0)}, E_{\gamma}^{(0)} & \text {, i.e. } \\
& E_{n}^{(0)}-E_{\alpha}^{(0)}, E_{n}^{(0)}-E_{\beta}^{(0)}, E_{n}^{(0)}-E_{\gamma}^{(0)}, \cdots
\end{aligned}
$$

As a rule the differences (23) enter the denominators of the perturbation terms, so there should be satisfied the relations

$$
\alpha \neq n, \beta \neq n, \gamma \neq n, \cdots
$$

etc.; see e.g. [8] for further details.

For $N>2$ numerous terms entering $S_{N}$ composed of (10), (23) and (24) can be submitted to infinite summations over the states indicated on the left of (24).

In practice the way of deriving the sets of $S_{N}$ terms necessary for the Schrödinger perturbation formalism indicated above becomes a complicated task. Concurrent methods, obtained mainly without inclusion of the time parameter, are given in [9]-[17]. The computational applications performed with the aid of these methods seem to not provide us with a complete formalism suitable for a large perturbation order $N$. One of the by-products of the present paper is to make the perturbation method for large $N$ to be more simple than before.

\section{Feynman's Time-Dependent Formalism Referred to the Schrödinger Perturbation Theory}

Feynman diagrams including the time variable became a well-known tool in representing the quantum phenomena of different kind [18] [19] [20]. They could be applied also in the case of the Schrödinger perturbation calculation. A fundamental difficulty of such a treatment comes from an enormous inflation of the number of diagrams which had to be considered in case of a large perturbation order $N$. For, according to the Feynman formalism, we should calculate and combine the results of

$$
P_{N}=(N-1) !
$$

diagrams in order to obtain the energy expression equivalent to the $S_{N}$ terms entering the Schrödinger theory.

It is evident that

$$
P_{N}=S_{N}
$$

for $N=1,2$, and 3, but already for $N=4$ we have

$$
P_{4}=6>S_{4}=5 \text {. }
$$

It is easy to check that for $N \gg 3$ we have $P_{N} \gg S_{N}$. For example for $N=20$ we obtain

$$
P_{20}=19 ! \cong 1.23 \times 10^{17}
$$

and

$$
S_{20}=1.77 \times 10^{9}
$$

Evidently the ratio 


$$
P_{N} / S_{N}
$$

increases systematically with $N$ tending to a huge number.

But the Feynman theory was based on a linear time scale represented by the interval given in formula (1). We demonstrate-in the remainder part of the paper-that a different kind of the time scale, namely that having a circular-like character, can lead precisely to the diagrams and, in consequence, the energy terms dictated by the Schrödinger perturbation calculus.

\section{Scale of Time Suitable for the Schrödinger Perturbation Formalism, Its Contraction Points and Side Loops}

Our idea is to replace a tedious calculation of the perturbation energy attained with the aid of solving the perturbed Schrödinger eigenequation by an immediate production of the perturbed energy terms due to an application of a suitable scale of time; see Figures 1-4.

According to Leibniz [21] [22] [23] time is an ordering parameter for the events occurring in the nature. A reference to the Leibniz concept of time as a merely successive order of things can be done also in connection of a discussion of the Mach's principle and the structure of dynamical theories [24] [25] [26].

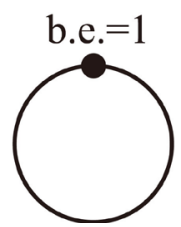

Figure 1. Time scale for the perturbation order $N=1$. The beginning-end point is 1 .

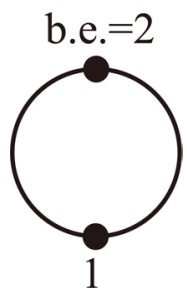

Figure 2. Time scale for the perturbation order $N=2$. Beyond of the beginning-end point 2 there exists also point 1 on the scale. No contraction between 1 and 2 is admissible.

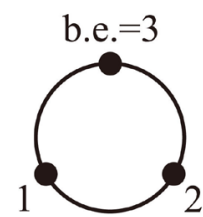

(a)

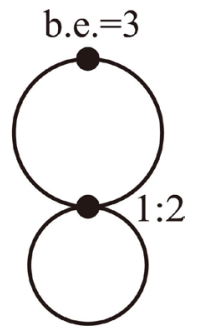

(b)

Figure 3. Time scale for the perturbation order $N=3$. Beyond of the beginning-end point 3 there exist also points 1 and 2 . They can remain either free [diagram (a)] or are contracted [diagram (b)] giving a side loop. 


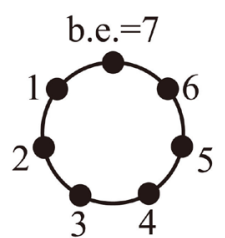

(a)

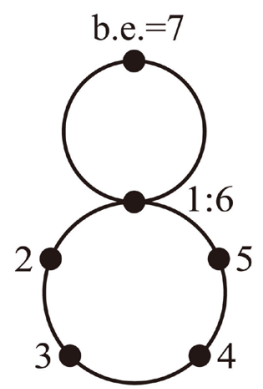

(b)

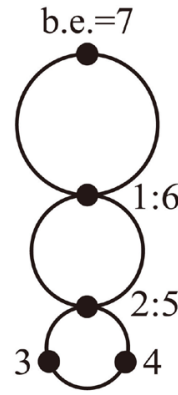

(c)

Figure 4. Time scale for the perturbation order $N=7$. Beyond of the beginning-end point 7 there exist also points 1, 2, 3, 4, 5 and 6 . They are free on the diagram (a), but can form-for example-a maximal side loop for $N=7$ due to contraction 1:6 [diagram (b)] or a cascade of loops [diagram (c)] due to contractions 1:6 and 2:5.

In case of the perturbation calculation, the Leibniz idea suggests to choose an appropriate scale of time, so it will be helpful to represent the results of the perturbation process. A necessary scale for any perturbation order $N$ occurs to be a circular-like scale. This implies that $N$ points of time-representing $N$ successive collisions of the quantum system with the perturbation potential (9)-are present on a topological circle. One of these points, say the $N$ th point, let be the beginning-end point of the scale, called henceforth the main scale, or loop, of time. The remainder $N-1$ time points on the scale can be left either free, or submitted to contractions.

The contractions of the time points done on the main scale lead to the side loop, or loops, of time. Since it occurs that the $N$ th point should be excluded from contractions, a maximal size of the loop created from the main loop of the $N$ points of time is given by the contraction between the time points 1 and $N-1$. This contraction is labelled by

$$
1: N-1 \text {. }
$$

Beyond of the maximal loop of (31), a set of the minimal loops due to contractions

$$
1: 2,2: 3,3: 4, \cdots, N-2: N-1
$$

can be also created. We can have still the intermediate side loops like

$$
1: 3,2: 4,3: 5, \cdots, N-3: N-1 \text {, }
$$

or other loops larger than those due to contractions in (33).

Beyond of single contractions listed in (31)-(33), also multiple contractions of the time points like

$$
1: 2: 3,2: 4: 5,2: 4: 7
$$

or

$$
2: 3: 5: 6,2: 4: 6: 7
$$

or those composed of a still larger number of the time points, should be taken into account: the size of the admitted contractions depends on the size of the 
considered $N$. Moreover, the combined contractions of the time points like

$$
1: 2 \bigcap 4: 5
$$

may come also into play. A general rule is that the time loops due to the acceptable contractions should not cross. This means that, for example, the combined contraction due to the pair

$$
1: 4 \cap 2: 5
$$

is not admissible.

A fundamental effect is that a full set of acceptable contractions for a considered order $N$ gives precisely the number $S_{N}$ of the Schrödinger perturbation terms predicted by the formula (21) for that $N$; no superflous neither lacking terms do occur. This is checked for the orders between $N=1$ and $N=7$ in the earlier papers by the author [25-34]. A full set of diagrams necessary for $N=6$ is given in [25], a similar set for $N=7$ enters [34]. In the present paper the perturbation energy of the order $N=8$ is also examined from the same point of view giving a similar agreement of the results; see Sec. 9 .

\section{Concentrations of the Contraction Points and Their Use}

The concentration of a contraction point is equal to the number of the loops of time which meet together in that point. Evidently, if the contraction point is located on the main loop of time, one of the loops met in that point is the main loop itself. The other loops created by the time contractions on the main loop are called the side loops. An advantage to operate with the concentrations of loops is that they allow us to express the perturbation results in a more compact form than could be expected before.

This is so because the concentrations which are characteristic for a given $N$ can be referred directly to partitions of the number $N-1$. In the next step the knowledge of partitions does lead to the number of the perturbation terms and the formulae for these terms. The effect of partitions and their connections with the contraction points will become evident in the computational practice giving the $S_{N}$ Schrödinger terms for $N=7$ and $N=8$; see Sec. 9 .

The next advantage of the time-point formalism is that any admissible contraction of the time points gives a correct contribution to the perturbation energy belonging to some $N$. The details of contributions can be easily derived by the analysis of contractions. The notation of the energy terms-see Sec. 6-can be simplified, because several terms of a given $N$ can combine into the expressions representing the perturbation energies belonging to $N^{\prime}<N$-the point usually neglected in the former perturbation calculations done by many authors. The way of calculation of particular energy expressions and examination of their properties are presented in Sec. 9, where the examples of $N=7$ and 8 are studied in detail. An evident effect due to such study is that the energy contribution given by any of the time-point contractions can be obtained. Details concerning such contributions and notation suitable to represent the perturbation terms are 
presented both in Sec. 6 and Sec. 9.

\section{Notation Applied to Represent the Energy Perturbation Terms}

Only for the perturbation orders $N=1$ and $N=2$ the side loops for the main loop of time do not exist. But any $S_{N}$ term for $N>2$ is a product of energy contributions due to the main loop of time and those due to the side loops, respectively.

The contributions due to the side loops are easy to access from contractions of the time points and will be discussed first. Any contraction

$$
\alpha: \beta
$$

where as a rule we have

$$
\alpha<\beta
$$

provide us with the energy multiplier equal to the energy correction

$$
\Delta E_{\beta-\alpha} \text {. }
$$

The difference

$$
\beta-\alpha
$$

indicates the perturbation order of energy contributed by the side loop represented by $\Delta E$. In result, when the difference indicated in (39) is larger than 2, we have more than one Schrödinger perturbation term represented by the side loop, for

$$
S_{\beta-\alpha}>1 \text {. }
$$

The contribution to energy due to the main loop of time depends on the number and situation of the time points present on that loop. When no contractions are present for the time points on the loop, the loop has $N$ time points on it and gives the energy term in the form

$$
\langle V P V P V P V P \cdots P V\rangle \text {. }
$$

Such loop carries $N$ symbols $V$ and $N-1$ symbols $P$.

Evidently for $N=1$ no $P$ symbol enters (41) and we obtain a single term for the perturbation energy equal to

$$
\langle V\rangle=\left\langle n\left|V^{\text {per }}\right| n\right\rangle=\Delta E_{1} .
$$

For the order $N=2$ we have no side loops and the perturbation energy is represented by the formula

$$
\langle V P V\rangle=\Delta E_{2}
$$

The symbol $P$ within the brackets on the left of (43) represents a reciprocal value of the energy difference, viz.

$$
P=\frac{1}{E_{n}^{(0)}-E_{p}^{(0)}},
$$

situated between two matrix elements of $V^{\text {per }}$, viz. 


$$
\left\langle n\left|V^{\text {per }}\right| p\right\rangle,\left\langle p\left|V^{\text {per }}\right| n\right\rangle,
$$

and submitted to summation process over the dummy state index $p$. In effect

$$
\langle V P V\rangle=\sum_{p \neq n} \frac{\left\langle n\left|V^{\mathrm{per}}\right| p\right\rangle\left\langle p\left|V^{\mathrm{per}}\right| n\right\rangle}{E_{n}^{(0)}-E_{p}^{(0)}} .
$$

The meaning similar to the term (45) does prolongate to any perturbation term given by the main loop of time carrying no contraction points. For example for $N=3$ such term is represented by

$$
\langle V P V P V\rangle=\sum_{p \neq n q \neq n} \frac{\left\langle n\left|V^{\mathrm{per}}\right| p\right\rangle\left\langle p\left|V^{\mathrm{per}}\right| q\right\rangle\left\langle q\left|V^{\mathrm{per}}\right| n\right\rangle}{\left[E_{n}^{(0)}-E_{p}^{(0)}\right]\left[E_{n}^{(0)}-E_{q}^{(0)}\right]} .
$$

This formula has two $P$ and two dummy indices ( $p$ and $q$ ) for summation over the quantum states with exclusion of state $n$ which is submitted to perturbation. It is easy to extend (46) to an arbitrary order $N$.

More complicated contributions to energy due to the main loop occur in case when the side loops are also present. For $N=3$ the only possible contraction of the time points is

$$
1: 2
$$

Evidently the side loop created by (47) does provide us with the term

$$
\langle V\rangle=\Delta E_{1},
$$

however our task is to present also a contribution due to the main loop of time. In this case contraction (47) transforms the term (46)-having no contractions-to the formula

$$
\left\langle V P^{2} V\right\rangle=\sum_{p \neq n} \frac{\left\langle n\left|V^{\mathrm{per}}\right| p\right\rangle\left\langle p\left|V^{\mathrm{per}}\right| n\right\rangle}{\left[E_{n}^{(0)}-E_{p}^{(0)}\right]^{2}} .
$$

The whole perturbation energy due to contraction (47) is represented by the product of (47a) and (48) taken with a minus sign:

$$
1: 2 \rightarrow-\left\langle V P^{2} V\right\rangle\langle V\rangle
$$

because we have an even number of terms entering the product in (49); an odd number of terms would give a positive sign. A characteristic point is that the total number of $P$ and $V$ entering the term in (49) remains the same as it does exist in the term (46): there are two $P$ and three $V$ together.

Another situation can be when the non-neighbouring time points, say 1 and 3 , enter contraction

$$
1: 3
$$

This may occur for the perturbation order equal at least to $N=4$, so the last time point 4 is the beginning-end point on the scale and does not enter into contractions.

The energy term for the main loop of time having no contraction points becomes - in this case-a triple sum of terms 


$$
\langle V P V P V P V\rangle=\sum_{p \neq n q \neq n \neq \neq n} \sum_{n} \frac{\left\langle n\left|V^{\mathrm{per}}\right| p\right\rangle\left\langle p\left|V^{\mathrm{per}}\right| q\right\rangle\left\langle q\left|V^{\mathrm{per}}\right| r\right\rangle\left\langle r\left|V^{\mathrm{per}}\right| n\right\rangle}{\left[E_{n}^{(0)}-E_{p}^{(0)}\right]\left[E_{n}^{(0)}-E_{q}^{(0)}\right]\left[E_{n}^{(0)}-E_{r}^{(0)}\right]}
$$

whereas the contraction (50) implies the side loop having point 2 as free on it. This makes the energy contribution due to the side loop equal to

$$
\langle V P V\rangle=\Delta E_{2}
$$

But the main loop of time having a contraction point (50) on it changes its contribution to the perturbation energy. Together with the beginning-end point of time the loop becomes similar to that representing the term (52), however the presence of the contraction point (50) implies the loop contribution to energy equal to

$$
\left\langle V P^{2} V\right\rangle=\sum_{p \neq n} \frac{\left\langle n\left|V^{\mathrm{per}}\right| p\right\rangle\left\langle p\left|V^{\mathrm{per}}\right| n\right\rangle}{\left[E_{n}^{(0)}-E_{p}^{(0)}\right]^{2}} .
$$

In effect the perturbation term due to contraction (50) is equal to product of (52) and (53):

$$
-\Delta E_{2}\left\langle V P^{2} V\right\rangle
$$

The minus sign in (54) is dictated by the presence of an even number of terms entering the final product.

The notation procedure indicated above can be extended to any perturbation order $N$.

\section{Time-Point Contractions on a Circular Scale and a Check of Validity of the Energy Terms Contributed by the Side-Loops of Time}

Let us begin with a maximal side loop presented by the time point contraction in (31). Because the number of free points of time present on the side loop in (31) is

$$
N^{\prime}=N-1-1=N-2,
$$

the energy contributed by the side loop due to (31) is equal to

$$
\Delta E_{N-2} \text {. }
$$

This energy has to be joined with the energy contribution given by the main loop of time which-due to contraction (31) - possess only two points of time: the beginning-end point and the contraction point (31). It should be noted that the presence of the beginning-end point does not give any contribution to the perturbation energy, for such contribution can be given only by a loop of time.

In effect of the contraction point of two loops, they are joined together. This implies that the energy term of the main loop should have the term

$$
P^{2}
$$

and not $P$ alone; see (53). But beyond of (57) we note that the main loop becomes similar to the time loop characteristic for the second-order perturbation 
term; see (52). In effect the main loop makes the whole contribution of the contraction (31) to the perturbation energy equal to

$$
-\left\langle V P^{2} V\right\rangle \Delta E_{N-2} \text {. }
$$

A formal check of validity of the energy expression given in (58) is simple: since the perturbation energy concerns order $N$, it should have the total number of $P$ in the perturbation expression equal to $N-1$ and the number $V$ is equal to $N$. Respectively, the perturbation energy in (51) contains the number of $P$ equal to $N-3$ and that of $V$ equal to $N-2$. The multiplier

$$
\left\langle V P^{2} V\right\rangle
$$

present in (58) supplies the lacking number of $P$ and $V$ in the term $\Delta E_{N-2}$ to the required number of $P$ and $V$ in an energy term belonging to $\Delta E_{N}$.

The same reasoning can be applied to any contraction of the time points

$$
1,2,3, \cdots, N-1
$$

entering the time scale useful for calculating the perturbation energy of the order $N$.

Examples of such calculations are presented in the earlier papers; see e.g. [33] [34].

The number of the time points which can be submitted to contractions for a given $N$ is $N-1$; evidently different contractions can give different concentrations at the contraction points. The point present on the scale having no contractions has concentration 1 , a maximal concentration of the $N-1$ time points is evidently $N-1$.

The same number $N-1$ is equal to the number of $P$ s present in any term of the Schrödinger perturbation energy; evidently for $N=1$ we have no $P$ present in the perturbation term.

\section{Systematic Time-Dependent Approach to the Schrödinger Perturbation Method. Partitions of the Number $N-1$ and the Time Point Contractions}

A fundametnal process of quantum mechanics is a change of a given system upon the action of a perturbation which-in its character-can be independent of time. To calculate the result of such a change on a non-degenerate system the Schrödinger perturbation formalism-represented by the sets of terms labelled by their order numbers $N$-is usually applied.

In principle there exist several ways according to which the necessary sets of terms can be obtained. In many cases no time approach should be used to this purpose. We are guided, however, by the Leibniz idea that a suitable arrangement of the perturbation events along a time scale can be helpful in an analysis of the expected change of a quantum system, including that due to the perturbation effect. Consequently the change of a system upon the action of a time-independent perturbation implies the importance of time. In fact we find that a circular scale of time-supplemented by the necessary time-point contractions on it-can represent with a perfect accuracy the perturbation terms of energy obtained in 
an almost automatic way.

All possible partitions of the number $N-1$ lead to respective time-point contractions necessary for calculating the perturbation terms belonging to that $N$. Certainly the time points entering contractions are dependent on the position of a given partition number in the sum equal to $N-1$. In this way we obtain a full set of necessary contractions for a given number $N-1$.

For example for $N=3$ we have $N-1=2$ and the only set of the acceptable contractions is reduced to a single contraction

$$
1: 2=N-2: N-1
$$

represented by a partition number of $N-1$ equal to 2 . But there exists also the partition

$$
11
$$

without contractions.

For $N=4$ we have the contractions $1: 2$ and $2: 3$ represented by partitions

$$
\begin{array}{ll}
2 & 1 \\
1 & 2
\end{array}
$$

Here the time point 3 and time point 1 remain free in the first and second row of (62), respectively. A full set of partitions for $N-1=3$ becomes

$$
\begin{array}{ccc}
1 & 1 & 1 \\
2 & 1 \\
1 & 2 \\
3 &
\end{array}
$$

the partition 3 does represent the contractions $1: 2: 3$ and $1: 3$.

For $N=5$ we obtain the partitions

$$
\begin{array}{rl}
111 & 1 \rightarrow \text { no contractions (points } 1,2,3,4 \text { free); } S_{1}^{4}=1 \\
21 & 1 \rightarrow 1: 2 \text {, points } 3,4 \text { free; } S_{2} S_{1}^{2}=1 \\
12 & 1 \rightarrow 2: 3 \text {, points } 1,4 \text { free; } S_{1} S_{2} S_{1}=1 \\
11 & 2 \rightarrow 3: 4 \text {, points } 1,2 \text { free; } S_{1}^{2} S_{2}=1 \\
2 & 2 \rightarrow 1: 2,3: 4 ; S_{1} S_{2}=1 \\
3 & 1 \rightarrow 1: 3,1: 2: 3 ; \text { point } 4 \text { free; } S_{3} S_{1}=2 \\
1 & \rightarrow \rightarrow 2: 4,2: 3: 4 ; \text { point } 1 \text { free; } S_{1} S_{3}=2 \\
& 4 \rightarrow 1: 4,1: 2: 4,1: 3: 4,1: 2: 3: 4,1: 4 \cap 2: 3 ; S_{4}=5 .
\end{array}
$$

A characteristic point is that the $S$-like results are equal to the number of contractions; an exception is the first term [see (64)] where the absence of contractions is associated with all partitions equal to 1 . A total value of the sum of the $S$-products on the left of (64)-(71) is equal to:

$$
\begin{aligned}
& S_{1}^{4}+S_{2} S_{1}^{2}+S_{1} S_{2} S_{1}+S_{1}^{2} S_{2}+S_{2}^{2}+S_{3} S_{1}+S_{1} S_{3}+S_{4} \\
& =1+1+1+1+1+2+2+5=14=S_{5} .
\end{aligned}
$$


Therefore a set of partitions of $N-1=4$ gives the $S_{5}$ perturbation terms.

A similar situation does repeat for $N=6$ which gives $N-1=5$; the partitions are

11111 (points $1,2,3,4,5$ free); $S_{1}^{5}=1$

$2111 \rightarrow 1: 2,(3,4,5$ free $) ; S_{2} S_{1}^{3}=1$

$1211 \rightarrow 2: 3,(1,4,5$ free $) ; S_{1} S_{2} S_{1}=1$

$1121 \rightarrow 3: 4,(1,2,5$ free $) ; S_{1}^{2} S_{2} S_{1}=1$

$1112 \rightarrow 4: 5,(1,2,3$ free $) ; S_{1}^{3} S_{2}=1$

$122 \rightarrow 2: 3 \bigcap 4: 5 ; S_{1} S_{2}^{2}=1$

$212 \rightarrow 1: 2 \bigcap 4: 5 ; S_{2} S_{1} S_{2}=1$

$221 \rightarrow 1: 2 \bigcap 3: 4 ; S_{2}^{2} S_{1}=1$

$113 \rightarrow 3: 5,3: 4: 5 ; S_{1}^{2} S_{3}=2$

$131 \rightarrow 2: 4,2: 3: 4 ; S_{1} S_{3} S_{1}=2$

$311 \rightarrow 1: 3,1: 2: 3 ; S_{3} S_{1}^{2}=2$

$41 \rightarrow 1: 4,1: 2: 4,1: 3: 4,1: 2: 3: 4,1: 4 \bigcap 2: 3 ; S_{4} S_{1}=5$

$14 \rightarrow 2: 5,2: 3: 5,2: 4: 5,2: 3: 4: 5,2: 5 \cap 3: 4 ; S_{1} S_{4}=5$

$5 \rightarrow 1: 5,1: 2: 5,1: 3: 5,1: 4: 5,1: 2: 3: 5,1: 3: 4: 5,1: 2: 3: 4: 5 ; S_{5}=14 ;(86)$

The total sum obtained from the $14 S$ like terms on the right is equal to

$$
\begin{aligned}
& S_{1}^{5}+S_{2} S_{1}^{3}+S_{1} S_{2} S_{1}^{2}+S_{1}^{2} S_{2} S_{1}+S_{1}^{3} S_{2}+S_{1} S_{2}^{2}+S_{2} S_{1} S_{2}+S_{2}^{2} S_{1} \\
& +S_{1}^{2} S_{3}+S_{1} S_{3} S_{1}+S_{3} S_{1}^{2}+S_{2} S_{3}+S_{3} S_{2}+S_{1} S_{4}+S_{4} S_{1}+S_{5} \\
& =1+1+1+1+1+1+1+1+2+2+2+2+2+5+5+14=42=S_{6} .
\end{aligned}
$$

Having the contraction data in (73)-(86) it becomes easy to construct the perturbation terms belonging to $N=6$. These terms are respectively:

$$
\begin{aligned}
& 11111 \rightarrow\langle V P V P V P V P V P V\rangle, \\
& 2111 \rightarrow-\left\langle V P^{2} V P V P V P V\right\rangle \Delta E_{1}, \\
& \left.\begin{array}{llll}
12 & 1 & 1 \rightarrow-\left\langle V P V P^{2} V P V P V\right\rangle
\end{array}\right\rangle E_{1}, \\
& \left.\begin{array}{llll}
11 & 2 & 1 \rightarrow-\left\langle V P V P V P^{2} V P V\right\rangle
\end{array}\right\rangle E_{1} \text {, } \\
& 1112 \rightarrow-\left\langle V P V P V P V P^{2} V\right\rangle \Delta E_{1}, \\
& 122 \rightarrow\left\langle V P V P^{2} V P^{2} V\right\rangle\left(\Delta E_{1}\right)^{2} \text {, } \\
& 212 \rightarrow\left\langle V P^{2} V P V P^{2} V\right\rangle\left(\Delta E_{1}\right)^{2} \text {, } \\
& 221 \rightarrow\left\langle V P^{2} V P^{2} V P V\right\rangle\left(\Delta E_{1}\right)^{2} \\
& 113 \rightarrow-\left\langle V P V P V P^{2} V\right\rangle \Delta E_{2} \text {, } \\
& \left\langle V P V P V P^{3} V\right\rangle\left(\Delta E_{1}\right)^{2},
\end{aligned}
$$




$$
\begin{aligned}
& 131 \rightarrow-\left\langle V P V P^{2} V P V\right\rangle \Delta E_{2}, \\
& \left\langle V P V P^{3} V P V\right\rangle\left(\Delta E_{1}\right)^{2}, \\
& 311 \rightarrow-\left\langle V P^{2} V P V P V\right\rangle \Delta E_{2}, \\
& \left\langle V P^{3} V P V P V\right\rangle\left(\Delta E_{1}\right)^{2}, \\
& 32 \rightarrow\left\langle V P^{2} V P^{2} V\right\rangle \Delta E_{2} \Delta E_{1} \text {, } \\
& -\left\langle V P^{3} V P^{2} V\right\rangle\left(\Delta E_{1}\right)^{2} \Delta E_{1}, \\
& 23 \rightarrow\left\langle V P^{2} V P^{2} V\right\rangle \Delta E_{1} \Delta E_{2} \text {, } \\
& -\left\langle V P^{2} V P^{3} V\right\rangle \Delta E_{1}\left(\Delta E_{1}\right)^{2}, \\
& 41 \rightarrow-\left\langle V P^{2} V P V\right\rangle \Delta E_{3} \text { (2) } \\
& \left\langle V P^{3} V P V\right\rangle \Delta E_{2} \Delta E_{1} \quad(1) \\
& \left\langle V P^{3} V P V\right\rangle \Delta E_{1} \Delta E_{2} \quad(1) \\
& -\left\langle V P^{4} V P V\right\rangle\left(\Delta E_{1}\right)^{3} \\
& 14 \rightarrow-\left\langle V P V P^{2} V\right\rangle \Delta E_{3} \\
& \left\langle V P V P^{3} V\right\rangle \Delta E_{2} \Delta E_{1} \quad(1) \\
& \left\langle V P V P^{3} V\right\rangle \Delta E_{1} \Delta E_{2}(1) \\
& -\left\langle V P V P^{4} V\right\rangle\left(\Delta E_{1}\right)^{3} \quad(1) \\
& 5 \rightarrow-\left\langle V P^{2} V\right\rangle \Delta E_{4} \text { (5) } \\
& \left\langle V P^{3} V\right\rangle \Delta E_{1} \Delta E_{3} \\
& \left\langle V P^{3} V\right\rangle\left(\Delta E_{2}\right)^{2} \\
& \left\langle V P^{3} V\right\rangle \Delta E_{3} \Delta E_{1} \\
& -\left\langle V P^{4} V\right\rangle\left(\Delta E_{1}\right)^{2} \Delta E_{2} \text { (1) } \\
& -\left\langle V P^{4} V\right\rangle \Delta E_{1} \Delta E_{2} \Delta E_{1} \quad(1) \\
& -\left\langle V P^{4} V\right\rangle \Delta E_{2}\left(\Delta E_{1}\right)^{2} \text { (1) } \\
& \left\langle V P^{5} V\right\rangle\left(\Delta E_{1}\right)^{4}
\end{aligned}
$$

The numbers in brackets represent the quantity of the perturbation terms in a given rows.

In a similar way the results for the perturbation terms belonging to $N=7$ and $N=8$ are obtained; the terms are represented in Tables 1-6.

\section{Comparison of the Present Method with an Earlier Recurrent Approach to the Perturbation Energy [34]}

In [34] we presented a formalism which makes a recurrent calculation of the Schrödinger perturbation energy possible for an arbitrary order $N$. The method-outlined in the present paper-is based on partitions of the number $N-1$. It seems to be more transparent and systematical than that given in [34].

The present Section compares the both approaches-that of [34] and that of 
Table 1. $N=7$. Perturbation terms based on the smaller size of partitions of the number $N-1=6$. Total number of the perturbation terms in the Table:

$(6)+(7)+(8)+(12)+(4)=(37)$.

\begin{tabular}{|c|c|c|}
\hline 111111 & $S_{1}^{6}=1 \rightarrow\langle V P V P V P V P V P V P V\rangle$ & $(1)$ \\
\hline 21111 & $S_{2} S_{1}^{4}=1 \rightarrow-\left\langle V P^{2} V P V P V P V P V\right\rangle \Delta E_{1}$ & (1) \\
\hline 12111 & $S_{2} S_{1}^{4}=1 \rightarrow-\left\langle V P V P^{2} V P V P V P V\right\rangle \Delta E_{1}$ & $(1)$ \\
\hline 11211 & $S_{2} S_{1}^{4}=1 \rightarrow-\left\langle V P V P V P^{2} V P V P V\right\rangle \Delta E_{1}$ & $(1)$ \\
\hline 11121 & $S_{2} S_{1}^{4}=1 \rightarrow-\left\langle V P V P V P V P^{2} V P V\right\rangle \Delta E_{1}$ & $(1)$ \\
\hline 11112 & $S_{2} S_{1}^{4}=1 \rightarrow-\left\langle V P V P V P V P V P^{2} V\right\rangle \Delta E_{1}$ & $(1)$ \\
\hline 2211 & $S_{2}^{2} S_{1}^{2}=1 \rightarrow\left\langle V P^{2} V P^{2} V P V P V\right\rangle\left(\Delta E_{1}\right)^{2}$ & $(1)$ \\
\hline 2121 & $S_{2}^{2} S_{1}^{2}=1 \rightarrow\left\langle V P^{2} V P V P^{2} V P V\right\rangle\left(\Delta E_{1}\right)^{2}$ & (1) \\
\hline 2112 & $S_{2}^{2} S_{1}^{2}=1 \rightarrow\left\langle V P^{2} V P V P V P^{2} V\right\rangle\left(\Delta E_{1}\right)^{2}$ & (1) \\
\hline 1221 & $S_{2}^{2} S_{1}^{2}=1 \rightarrow\left\langle V P V P^{2} V P^{2} V P V\right\rangle\left(\Delta E_{1}\right)^{2}$ & $(1)$ \\
\hline 1212 & $S_{2}^{2} S_{1}^{2}=1 \rightarrow\left\langle V P V P^{2} V P V P^{2} V\right\rangle\left(\Delta E_{1}\right)^{2}$ & $(1)$ \\
\hline 1122 & $S_{2}^{2} S_{1}^{2}=1 \rightarrow\left\langle V P V P V P^{2} V P^{2} V\right\rangle\left(\Delta E_{1}\right)^{2}$ & (1) \\
\hline 222 & $S_{2}^{3}=1 \rightarrow-\left\langle V P^{2} V P^{2} V P^{2} V\right\rangle\left(\Delta E_{1}\right)^{3}$ & $(1)$ \\
\hline \multirow[t]{2}{*}{3111} & $S_{3} S_{1}^{3}=2 \rightarrow-\left\langle V P^{2} V P V P V P V\right\rangle \Delta E_{2}$ & $(1)$ \\
\hline & $\rightarrow\left\langle V P^{3} V P V P V P V\right\rangle\left(\Delta E_{1}\right)^{2}$ & (1) \\
\hline \multirow[t]{2}{*}{1311} & $S_{3} S_{1}^{3}=2 \rightarrow-\left\langle V P V P^{2} V P V P V\right\rangle \Delta E_{2}$ & $(1)$ \\
\hline & $\rightarrow\left\langle V P V P^{3} V P V P V\right\rangle\left(\Delta E_{1}\right)^{2}$ & $(1)$ \\
\hline \multirow[t]{2}{*}{1131} & $S_{1}^{3} S_{3}=2 \rightarrow-\left\langle V P V P V P^{2} V P V\right\rangle \Delta E_{2}$ & $(1)$ \\
\hline & $\rightarrow\left\langle V P V P V P^{3} V P V\right\rangle\left(\Delta E_{1}\right)^{2}$ & (1) \\
\hline \multirow[t]{2}{*}{1113} & $S_{1}^{3} S_{3}=2 \rightarrow-\left\langle V P V P V P V P^{2} V\right\rangle \Delta E_{2}$ & $(1)$ \\
\hline & $\rightarrow\left\langle V P V P V P V P^{3} V\right\rangle\left(\Delta E_{1}\right)^{2}$ & (1) \\
\hline \multirow[t]{2}{*}{321} & $S_{3} S_{2} S_{1}=2 \rightarrow\left\langle V P^{2} V P^{2} V P V\right\rangle \Delta E_{2} \Delta E_{1}$ & (1) \\
\hline & $\rightarrow\left\langle-V P^{3} V P^{2} V P V\right\rangle\left(\Delta E_{1}\right)^{3}$ & $(1)$ \\
\hline \multirow[t]{2}{*}{312} & $S_{3} S_{1} S_{2}=2 \rightarrow\left\langle V P^{2} V P V P^{2} V\right\rangle \Delta E_{2} \Delta E_{1}$ & $(1)$ \\
\hline & $\rightarrow-\left\langle V P^{3} V P V P^{2} V\right\rangle\left(\Delta E_{1}\right)^{3}$ & $(1)$ \\
\hline \multirow[t]{2}{*}{231} & $S_{2} S_{3} S_{1}=2 \rightarrow\left\langle V P^{2} V P^{2} V P V\right\rangle \Delta E_{1} \Delta E_{2}$ & (1) \\
\hline & $\rightarrow-\left\langle V P^{2} V P^{3} V P V\right\rangle\left(\Delta E_{1}\right)^{3}$ & (1) \\
\hline \multirow[t]{2}{*}{213} & $S_{2} S_{1} S_{3}=2 \rightarrow\left\langle V P^{2} V P V P^{2} V\right\rangle \Delta E_{1} \Delta E_{2}$ & $(1)$ \\
\hline & $\rightarrow-\left\langle V P V P V P^{3} V\right\rangle\left(\Delta E_{1}\right)^{3}$ & $(1)$ \\
\hline
\end{tabular}




\section{Continued}

$\begin{aligned} & S_{1} S_{3} S_{2}=2 \rightarrow\left\langle V P V P^{2} V P^{2} V\right\rangle \Delta E_{2} \Delta E_{1} \\ & \rightarrow-\left\langle V P V P^{3} V P^{2} V\right\rangle\left(\Delta E_{1}\right)^{3} \\ & S_{1} S_{2} S_{3}=2 \rightarrow\left\langle V P V P^{2} V P^{2} V\right\rangle \Delta E_{1} \Delta E_{2} \\ & \rightarrow-\left\langle V P V P^{2} V P^{3} V\right\rangle\left(\Delta E_{1}\right)^{3} \\ & S_{3} S_{3}=4 \rightarrow\left\langle V P^{2} V P^{2} V\right\rangle\left(\Delta E_{2}\right)^{2} \\ & \rightarrow-\left\langle V P^{3} V P^{2} V\right\rangle\left(\Delta E_{1}\right)^{2} \Delta E_{2} \\ & \rightarrow-\left\langle V P^{2} V P^{3} V\right\rangle \Delta E_{2}\left(\Delta E_{1}\right)^{2} \\ & \rightarrow\left\langle V P^{3} V P^{3} V\right\rangle\left(\Delta E_{1}\right)^{4}\end{aligned}$

Table 2. $N=7$. Perturbation terms based on the intermediate size of partitions of the number $N-1=6$. Total number of the perturbation terms:

$(5)+(5)+(5)+(14)+(14)=(53)$.

411

$S_{4} S_{1}^{2}=5 \rightarrow-\left\langle V P^{2} V P V P V\right\rangle \Delta E_{3}$

$\rightarrow\left\langle V P^{3} V P V P V\right\rangle \Delta E_{1} \Delta E_{2}$

$\rightarrow\left\langle V P^{3} V P V P V\right\rangle \Delta E_{2} \Delta E_{1}$

$\rightarrow-\left\langle V P^{4} V P V P V\right\rangle\left(\Delta E_{1}\right)^{3}$

141

$S_{1}^{2} S_{4}=5 \rightarrow-\left\langle V P V P^{2} V P V\right\rangle \Delta E_{3}$

$\rightarrow\left\langle V P V P^{3} V P V\right\rangle \Delta E_{1} \Delta E_{2}$

$\rightarrow\left\langle V P V P^{3} V P V\right\rangle \Delta E_{2} \Delta E_{1}$

$\rightarrow-\left\langle V P V P^{4} V P V\right\rangle\left(\Delta E_{1}\right)^{3}$

114

$S_{1}^{2} S_{4}=5 \rightarrow-\left\langle V P V P V P^{2} V\right\rangle \Delta E_{3}$

$\rightarrow\left\langle V P V P V P^{3} V\right\rangle \Delta E_{1} \Delta E_{2}$

$\rightarrow\left\langle V P V P V P^{3} V\right\rangle \Delta E_{2} \Delta E_{1}$

$\rightarrow-\left\langle V P V P V P^{4} V\right\rangle\left(\Delta E_{1}\right)^{3}$

42

$S_{4} S_{2}=5 \rightarrow\left\langle V P^{2} V P^{2} V\right\rangle \Delta E_{3} \Delta E_{1}$

$\rightarrow-\left\langle V P^{3} V P^{2} V\right\rangle\left(\Delta E_{1}\right)^{2} \Delta E_{2}$

$\rightarrow-\left\langle V P^{3} V P^{2} V\right\rangle \Delta E_{1} \Delta E_{2} \Delta E_{1}$

$\rightarrow\left\langle V P^{4} V P^{2} V\right\rangle\left(\Delta E_{1}\right)^{4}$

24

$S_{2} S_{4}=5 \rightarrow\left\langle V P^{2} V P^{2} V\right\rangle \Delta E_{1} \Delta E_{3}$

$\rightarrow-\left\langle V P^{2} V P^{3} V\right\rangle \Delta E_{1} \Delta E_{2} \Delta E_{3}$

$\rightarrow-\left\langle V P^{2} V P^{3} V\right\rangle \Delta E_{2}\left(\Delta E_{1}\right)^{2}$ 


\section{Continued}

$\rightarrow\left\langle V P^{2} V P^{4} V\right\rangle\left(\Delta E_{1}\right)^{4}$

51

$S_{5} S_{1}=14 \rightarrow-\left\langle V P^{2} V P V\right\rangle \Delta E_{4}$

$\rightarrow\left\langle V P^{3} V P V\right\rangle \Delta E_{3} \Delta E_{1}$

$\rightarrow\left\langle V P^{3} V P V\right\rangle\left(\Delta E_{2}\right)^{2}$

$\rightarrow\left\langle V P^{3} V P V\right\rangle \Delta E_{1} \Delta E_{3}$

$\rightarrow-\left\langle V P^{4} V P V\right\rangle\left(\Delta E_{1}\right)^{2} \Delta E_{2}$

$\rightarrow-\left\langle V P^{4} V P V\right\rangle \Delta E_{1} \Delta E_{2} \Delta E_{1}$

$\rightarrow-\left\langle V P^{4} V P V\right\rangle \Delta E_{2}\left(\Delta E_{1}\right)^{2}$

$\rightarrow\left\langle V P^{5} V P V\right\rangle\left(\Delta E_{1}\right)^{4}$

15

$S_{1} S_{5}=14 \rightarrow-\left\langle V P V P^{2} V\right\rangle \Delta E$

$\rightarrow\left\langle V P V P^{3} V\right\rangle \Delta E_{3} \Delta E_{1}$

$\rightarrow\left\langle V P V P^{3} V\right\rangle\left(\Delta E_{2}\right)^{2}$

$\rightarrow\left\langle V P V P^{3} V\right\rangle \Delta E_{1} \Delta E_{3}$

$\rightarrow-\left\langle V P V P^{4} V\right\rangle\left(\Delta E_{1}\right)^{2} \Delta E_{2}$

$\rightarrow-\left\langle V P V P^{4} V\right\rangle \Delta E_{1} \Delta E_{2} \Delta E_{1}$

$\rightarrow-\left\langle V P V P^{4} V\right\rangle \Delta E_{2}\left(\Delta E_{1}\right)^{2}$

$\rightarrow\left\langle V P V P^{5} V\right\rangle\left(\Delta E_{1}\right)^{4}$

Table 3. $N=7$. The 42 energy perturbation terms belonging to partition $6=N-1$. The time-point contractions applied in the Table are presented. The number in brackets at the end of each row indicates the number of the perturbation terms due to that row. Total number of terms in Tables 1-3: $(37)+(53)+(42)=132=S_{7}$.

$\begin{array}{cll}1: 6 & \rightarrow-\left\langle V P^{2} V\right\rangle \Delta E_{5} & \rightarrow S_{5}=(14) \\ 1: 2: 6 & \rightarrow\left\langle V P^{3} V\right\rangle \Delta E_{1} \Delta E_{4} & \rightarrow S_{1} S_{4}=(5) \\ 1: 3: 6 & \rightarrow\left\langle V P^{3} V\right\rangle \Delta E_{2} \Delta E_{3} & \rightarrow S_{2} S_{3}=(2) \\ 1: 4: 6 & \rightarrow\left\langle V P^{3} V\right\rangle \Delta E_{3} \Delta E_{2} & \rightarrow S_{3} S_{2}=(2) \\ 1: 5: 6 & \rightarrow\left\langle V P^{3} V\right\rangle \Delta E_{4} \Delta E_{1} & \rightarrow S_{4} S_{1}=(5) \\ 1: 2: 3: 6 & \rightarrow-\left\langle V P^{4} V\right\rangle\left(\Delta E_{1}\right)^{2} \Delta E_{3} & \rightarrow S_{1}^{2} S_{3}=(2) \\ 1: 2: 4: 6 & \rightarrow-\left\langle V P^{4} V\right\rangle \Delta E_{1}\left(\Delta E_{2}\right)^{2} & \rightarrow S_{1} S_{2}^{2}=(1) \\ 1: 2: 5: 6 & \rightarrow-\left\langle V P^{4} V\right\rangle \Delta E_{1} \Delta E_{3} \Delta E_{1} & \rightarrow S_{1}^{2} S_{3}^{2}=(2) \\ 1: 3: 4: 6 & \rightarrow-\left\langle V P^{4} V\right\rangle \Delta E_{2} \Delta E_{1} \Delta E_{2} & \rightarrow S_{2}^{2} S_{1}^{2}=(1)\end{array}$




\section{Continued}

\begin{tabular}{cll}
\hline $1: 3: 5: 6$ & $\rightarrow-\left\langle V P^{4} V\right\rangle\left(\Delta E_{2}\right)^{2} \Delta E_{1}$ & $\rightarrow S_{2}^{2} S_{1}^{2}=(1)$ \\
$1: 4: 5: 6$ & $\rightarrow-\left\langle V P^{4} V\right\rangle \Delta E_{3}\left(\Delta E_{1}\right)^{2}$ & $\rightarrow S_{3} S_{1}^{2}=(2)$ \\
$1: 2: 3: 4: 6$ & $\rightarrow\left\langle V P^{5} V\right\rangle\left(\Delta E_{1}\right)^{3} \Delta E_{2}$ & $\rightarrow S_{1}^{3} S_{2}=(1)$ \\
$1: 2: 3: 5: 6$ & $\rightarrow\left\langle V P^{5} V\right\rangle\left(\Delta E_{1}\right)^{2} \Delta E_{2} \Delta E_{1}$ & $\rightarrow S_{1}^{3} S_{2}=(1)$ \\
$1: 2: 4: 5: 6$ & $\rightarrow\left\langle V P^{5} V\right\rangle \Delta E_{1} \Delta E_{2}\left(\Delta E_{1}\right)^{2}$ & $\rightarrow S_{1}^{3} S_{2}=(1)$ \\
$1: 3: 4: 5: 6$ & $\rightarrow\left\langle V P^{5} V\right\rangle \Delta E_{2}\left(\Delta E_{1}\right)^{3}$ & $\rightarrow S_{1}^{3} S_{2}=(1)$ \\
$1: 2: 3: 4: 5: 6$ & $\rightarrow-\left\langle V P^{6} V\right\rangle\left(\Delta E_{1}\right)^{5}$ & $\rightarrow S_{1}^{5}=(1)$
\end{tabular}

Table 4. $N=8$. Perturbation terms based on the lower-size partitions of the number $N-1=7$. Total number of the perturbation terms represented in Table 4:

$(7)+(10)+(4)+(10)+(24)+(6)+(12)+4 \times(5)=(93)$.

\begin{tabular}{|c|c|c|}
\hline 1111111 & $S_{1}^{7}=1 \rightarrow\langle V P V P V P V P V P V P V P V\rangle$ & (1) \\
\hline 211111 & $S_{2} S_{1}^{5}=1 \rightarrow-\left\langle V P^{2} V P V P V P V P V P V\right\rangle \Delta E_{1}$ & (1) \\
\hline 121111 & $S_{2} S_{1}^{5}=1 \rightarrow-\left\langle V P V P^{2} V P V P V P V P V\right\rangle \Delta E_{1}$ & (1) \\
\hline 112111 & $S_{2} S_{1}^{5}=1 \rightarrow-\left\langle V P V P V P^{2} V P V P V P V\right\rangle \Delta E_{1}$ & (1) \\
\hline 111211 & $S_{2} S_{1}^{5}=1 \rightarrow-\left\langle V P V P V P V P^{2} V P V P V\right\rangle \Delta E_{1}$ & (1) \\
\hline 111121 & $S_{2} S_{1}^{5}=1 \rightarrow-\left\langle V P V P V P V P V P^{2} V P V\right\rangle \Delta E_{1}$ & (1) \\
\hline 111112 & $S_{2} S_{1}^{5}=1 \rightarrow-\left\langle V P V P V P V P V P V P^{2} V\right\rangle \Delta E_{1}$ & (1) \\
\hline 22111 & $S_{2}^{2} S_{1}^{3}=1 \rightarrow\left\langle V P^{2} V P^{2} V P V P V P V\right\rangle\left(\Delta E_{1}\right)^{2}$ & (1) \\
\hline 12112 & $S_{1}^{3} S_{2}^{2}=1 \rightarrow\left\langle V P V P^{2} V P V P V P^{2} V\right\rangle\left(\Delta E_{1}\right)^{2}$ & (1) \\
\hline 11212 & $S_{1}^{3} S_{2}^{2}=1 \rightarrow\left\langle V P V P V P^{2} V P V P^{2} V\right\rangle\left(\Delta E_{1}\right)^{2}$ & (1) \\
\hline 11122 & $S_{1}^{3} S_{2}^{2}=1 \rightarrow\left\langle V P V P V P V P^{2} V P^{2} V\right\rangle\left(\Delta E_{1}\right)^{2}$ & $(1)$ \\
\hline 21112 & $S_{1}^{3} S_{2}^{2}=1 \rightarrow\left\langle V P^{2} V P V P V P V P^{2} V\right\rangle\left(\Delta E_{1}\right)^{2}$ & (1) \\
\hline 21211 & $S_{2}^{2} S_{1}^{3}=1 \rightarrow\left\langle V P^{2} V P V P^{2} V P V P V\right\rangle\left(\Delta E_{1}\right)^{2}$ & $(1)$ \\
\hline 21121 & $S_{2}^{2} S_{1}^{3}=1 \rightarrow\left\langle V P^{2} V P V P V P^{2} V P V\right\rangle\left(\Delta E_{1}\right)^{2}$ & $(1)$ \\
\hline 12211 & $S_{2}^{2} S_{1}^{3}=1 \rightarrow\left\langle V P V P^{2} V P^{2} V P V P V\right\rangle\left(\Delta E_{1}\right)^{2}$ & $(1)$ \\
\hline 11221 & $S_{1}^{3} S_{2}^{2}=1 \rightarrow\left\langle V P V P V P^{2} V P^{2} V P V\right\rangle\left(\Delta E_{1}\right)^{2}$ & $(1)$ \\
\hline 12121 & $S_{1}^{3} S_{2}^{2}=1 \rightarrow\left\langle V P V P^{2} V P V P^{2} V P V\right\rangle\left(\Delta E_{1}\right)^{2}$ & $(1)$ \\
\hline 2221 & $S_{2}^{3} S_{1}=1 \rightarrow-\left\langle V P^{2} V P^{2} V P^{2} V P V\right\rangle\left(\Delta E_{1}\right)^{3}$ & (1) \\
\hline 2212 & $S_{2}^{3} S_{1}=1 \rightarrow-\left\langle V P^{2} V P^{2} V P V P^{2} V\right\rangle\left(\Delta E_{1}\right)^{3}$ & (1) \\
\hline 2122 & $S_{2}^{3} S_{1}=1 \rightarrow-\left\langle V P^{2} V P V P^{2} V P^{2} V\right\rangle\left(\Delta E_{1}\right)^{3}$ & $(1)$ \\
\hline
\end{tabular}




\section{Continued}

\begin{tabular}{|c|c|c|}
\hline 1222 & $S_{1} S_{2}^{3}=1 \rightarrow-\left\langle V P V P^{2} V P^{2} V P^{2} V\right\rangle\left(\Delta E_{1}\right)^{3}$ & (1) \\
\hline 31111 & $\begin{array}{l}S_{3} S_{1}^{4}=2 \rightarrow-\left\langle V P^{2} V P V P V P V P V\right\rangle \Delta E_{2} \\
\rightarrow\left\langle V P^{3} V P V P V P V P V\right\rangle\left(\Delta E_{1}\right)^{2}\end{array}$ & (2) \\
\hline 13111 & $\begin{array}{l}S_{1}^{4} S_{3}=2 \rightarrow-\left\langle V P V P^{2} V P V P V P V\right\rangle \Delta E_{2} \\
\rightarrow\left\langle V P V P^{3} V P V P V P V\right\rangle\left(\Delta E_{1}\right)^{2}\end{array}$ & (2) \\
\hline 11311 & $\begin{array}{l}S_{1}^{4} S_{3}=2 \rightarrow-\left\langle V P V P V P^{2} V P V P V\right\rangle \Delta E_{2} \\
\rightarrow\left\langle V P V P V P^{3} V P V P V\right\rangle\left(\Delta E_{1}\right)^{2}\end{array}$ & (2) \\
\hline 11131 & $\begin{array}{l}S_{1}^{4} S_{3}=2 \rightarrow-\left\langle V P V P V P V P^{2} V P V\right\rangle \Delta E_{2} \\
\rightarrow\left\langle V P V P V P V P^{3} V P V\right\rangle\left(\Delta E_{1}\right)^{2}\end{array}$ & (2) \\
\hline 11113 & $\begin{array}{l}S_{1}^{4} S_{3}=2 \rightarrow-\left\langle V P V P V P V P V P^{2} V\right\rangle \Delta E_{2} \\
\rightarrow\left\langle V P V P V P V P V P^{3} V\right\rangle\left(\Delta E_{1}\right)^{2}\end{array}$ & (2) \\
\hline 3211 & $\begin{array}{l}S_{3} S_{2} S_{1}^{2}=2 \rightarrow\left\langle V P^{2} V P^{2} V P V P V\right\rangle \Delta E_{2} \Delta E_{1} \\
\rightarrow-\left\langle V P^{3} V P^{2} V P V P V\right\rangle\left(\Delta E_{1}\right)^{3}\end{array}$ & (2) \\
\hline 1321 & $\begin{array}{l}S_{1}^{2} S_{3} S_{2}=2 \rightarrow\left\langle V P V P^{2} V P^{2} V P V\right\rangle \Delta E_{2} \Delta E_{1} \\
\rightarrow-\left\langle V P V P^{3} V P^{2} V P V\right\rangle\left(\Delta E_{1}\right)^{3}\end{array}$ & (2) \\
\hline 2131 & $\begin{array}{l}S_{2} S_{1}^{2} S_{3}=2 \rightarrow\left\langle V P^{2} V P V P^{2} V P V\right\rangle \Delta E_{1} \Delta E_{2} \\
\rightarrow-\left\langle V P^{2} V P V P^{3} V P V\right\rangle\left(\Delta E_{1}\right)^{3}\end{array}$ & (2) \\
\hline 3121 & $\begin{array}{l}S_{3} S_{1}^{2} S_{2}=2 \rightarrow\left\langle V P^{2} V P V P^{2} V P V\right\rangle \Delta E_{2} \Delta E_{1} \\
\rightarrow-\left\langle V P^{3} V P V P^{2} V P V\right\rangle\left(\Delta E_{1}\right)^{3}\end{array}$ & (2) \\
\hline 2311 & $\begin{array}{l}S_{2} S_{3} S_{1}^{2}=2 \rightarrow\left\langle V P^{2} V P^{2} V P V P V\right\rangle \Delta E_{1} \Delta E_{2} \\
\rightarrow-\left\langle V P^{2} V P^{3} V P V P V\right\rangle\left(\Delta E_{1}\right)^{3}\end{array}$ & (2) \\
\hline 1231 & $\begin{array}{l}S_{1}^{2} S_{2} S_{3}=2 \rightarrow\left\langle V P V P^{2} V P^{2} V P V\right\rangle \Delta E_{1} \Delta E_{2} \\
\rightarrow-\left\langle V P V P^{2} V P^{3} V P V\right\rangle\left(\Delta E_{1}\right)^{3}\end{array}$ & (2) \\
\hline 1123 & $\begin{array}{l}S_{1}^{2} S_{2} S_{3}=2 \rightarrow\left\langle V P V P V P^{2} V P^{2} V\right\rangle \Delta E_{1} \Delta E_{2} \\
\rightarrow-\left\langle V P V P V P^{2} V P^{3} V\right\rangle\left(\Delta E_{1}\right)^{3}\end{array}$ & (2) \\
\hline 1132 & $\begin{array}{l}S_{1}^{2} S_{3} S_{2}=2 \rightarrow\left\langle V P V P V P^{2} V P^{2} V\right\rangle \Delta E_{2} \Delta E_{1} \\
\rightarrow-\left\langle V P V P V P^{3} V P^{2} V\right\rangle\left(\Delta E_{1}\right)^{3}\end{array}$ & (2) \\
\hline 1213 & $\begin{array}{l}S_{1}^{2} S_{2} S_{3}=2 \rightarrow\left\langle V P V P^{2} V P V P^{2} V\right\rangle \Delta E_{1} \Delta E_{2} \\
\rightarrow-\left\langle V P V P^{2} V P V P^{3} V\right\rangle\left(\Delta E_{1}\right)^{3}\end{array}$ & (2) \\
\hline
\end{tabular}




\section{Continued}

1312

2113

3112

322

232

223

331

133

4111

411
$S_{1}^{2} S_{3} S_{2}=2 \rightarrow\left\langle V P V P^{2} V P V P^{2} V\right\rangle \Delta E_{1} \Delta E_{2}$

$\rightarrow-\left\langle V P V P^{3} V P V P^{2} V\right\rangle\left(\Delta E_{1}\right)^{3}$

$S_{2} S_{1}^{2} S_{3}=2 \rightarrow\left\langle V P^{2} V P V P V P^{2} V\right\rangle \Delta E_{1} \Delta E_{2}$

$\rightarrow-\left\langle V P^{2} V P V P V P^{3} V\right\rangle\left(\Delta E_{1}\right)^{3}$

$S_{3} S_{1}^{2} S_{2}=2 \rightarrow\left\langle V P^{2} V P V P V P^{2} V\right\rangle \Delta E_{2} \Delta E_{1}$

$\rightarrow-\left\langle V P^{3} V P V P V P^{2} V\right\rangle\left(\Delta E_{1}\right)^{3}$

$\rightarrow\left\langle V P^{3} V P^{2} V P^{2} V\right\rangle\left(\Delta E_{1}\right)^{4}$

$S_{2}^{2} S_{3}=2 \rightarrow-\left\langle V P^{2} V P^{2} V P^{2} V\right\rangle \Delta E_{1} \Delta E_{2} \Delta E_{1}$

$\rightarrow\left\langle V P^{2} V P^{3} V P^{2} V\right\rangle\left(\Delta E_{1}\right)^{4}$

$S_{2}^{2} S_{3}=2 \rightarrow-\left\langle V P^{2} V P^{2} V P^{2} V\right\rangle\left(\Delta E_{1}\right)^{2} \Delta E_{2}$

$\rightarrow\left\langle V P^{2} V P^{2} V P^{3} V\right\rangle\left(\Delta E_{1}\right)^{4}$

$S_{3}^{2} S_{1}=4 \rightarrow\left\langle V P^{2} V P^{2} V P V\right\rangle\left(\Delta E_{2}\right)^{2}$

$\rightarrow-\left\langle V P^{3} V P^{2} V P V\right\rangle\left(\Delta E_{1}\right)^{2} \Delta E_{2}$

$\rightarrow-\left\langle V P^{2} V P^{3} V P V\right\rangle \Delta E_{2}\left(\Delta E_{1}\right)^{2}$

$\rightarrow\left\langle V P^{3} V P^{3} V P V\right\rangle\left(\Delta E_{1}\right)^{4}$

$S_{3}^{2} S_{1}=4 \rightarrow\left\langle V P^{2} V P V P^{2} V\right\rangle\left(\Delta E_{2}\right)^{2}$

$\rightarrow-\left\langle V P^{3} V P V P^{2} V\right\rangle\left(\Delta E_{1}\right)^{2} \Delta E_{2}$

$\rightarrow\left\langle-V P^{2} V P V P^{3} V\right\rangle \Delta E_{2}\left(\Delta E_{1}\right)^{2}$

$\rightarrow\left\langle V P^{3} V P V P^{3} V\right\rangle\left(\Delta E_{1}\right)^{4}$

$S_{1} S_{3}^{2}=4 \rightarrow\left\langle V P V P^{2} V P^{2} V\right\rangle\left(\Delta E_{2}\right)^{2}$

$\rightarrow-\left\langle V P V P^{3} V P^{2} V\right\rangle\left(\Delta E_{1}\right)^{2} \Delta E_{2}$

$\rightarrow-\left\langle V P V P^{2} V P^{3} V\right\rangle \Delta E_{2}\left(\Delta E_{1}\right)^{2}$

$\rightarrow\left\langle V P V P^{3} V P^{3} V\right\rangle\left(\Delta E_{1}\right)^{4}$

$S_{4} S_{1}^{3}=5 \rightarrow-\left\langle V P^{2} V P V P V P V\right\rangle \Delta E_{3}$

$\rightarrow\left\langle V P^{3} V P V P V P V\right\rangle \Delta E_{1} \Delta E_{2}$

$\rightarrow\left\langle V P^{3} V P V P V P V\right\rangle \Delta E_{2} \Delta E_{1}$

$\rightarrow-\left\langle V P^{4} V P V P V P V\right\rangle\left(\Delta E_{1}\right)^{3}$

$S_{1}^{3} S_{4}=5 \rightarrow-\left\langle V P V P^{2} V P V P V\right\rangle \Delta E_{3}$

$\rightarrow\left\langle V P V P^{3} V P V P V\right\rangle \Delta E_{1} \Delta E_{2}$ 


\section{Continued}

$\rightarrow\left\langle V P V P^{3} V P V P V\right\rangle \Delta E_{2} \Delta E_{1}$

$\rightarrow-\left\langle V P V P^{4} V P V P V\right\rangle\left(\Delta E_{1}\right)^{3}$

1141

$S_{1}^{3} S_{4}=5 \rightarrow-\left\langle V P V P V P^{2} V P V\right\rangle \Delta E_{3}$

$\rightarrow\left\langle V P V P V P^{3} V P V\right\rangle \triangle E_{1} \Delta E_{2}$

$\rightarrow\left\langle V P V P V P^{3} V P V\right\rangle \Delta E_{2} \Delta E_{1}$

$\rightarrow-\left\langle V P V P V P^{4} V P V\right\rangle\left(\Delta E_{1}\right)^{3}$

1114

$S_{1}^{3} S_{4}=5 \rightarrow-\left\langle V P V P V P V P^{2} V\right\rangle \Delta E_{3}$

$\rightarrow\left\langle V P V P V P V P^{3} V\right\rangle \Delta E_{1} \Delta E_{2}$

$\rightarrow\left\langle V P V P V P V P^{3} V\right\rangle \Delta E_{2} \Delta E_{1}$

$\rightarrow-\left\langle V P V P V P V P^{4} V\right\rangle\left(\Delta E_{1}\right)^{3}$

Table 5. $N=8$. Perturbation terms based on the higher-size partitions of the number $N-1=7$. Total number of the perturbation terms:

$6 \times(5)+2 \times(10)+2 \times(14)+3 \times(14)+2 \times(42)=(204)$.

421

$S_{4} S_{2} S_{1}=5 \rightarrow\left\langle V P^{2} V P^{2} V P V\right\rangle \Delta E_{3} \Delta E_{1}$

$\rightarrow-\left\langle V P^{3} V P^{2} V P V\right\rangle \Delta E_{2}\left(\Delta E_{1}\right)^{2}$

$\rightarrow-\left\langle V P^{3} V P^{2} V P V\right\rangle \Delta E_{1} \Delta E_{2} \Delta E_{1}$

$\rightarrow\left\langle V P^{4} V P^{2} V P V\right\rangle\left(\Delta E_{1}\right)^{2}$

412

$S_{4} S_{1} S_{2}=5 \rightarrow\left\langle V P^{2} V P V P^{2} V\right\rangle \Delta E_{3} \Delta E_{1}$

$\rightarrow-\left\langle V P^{3} V P V P^{2} V\right\rangle \Delta E_{2}\left(\Delta E_{1}\right)^{2}$

$\rightarrow-\left\langle V P^{3} V P V P^{2} V\right\rangle \Delta E_{1} \Delta E_{2} \Delta E_{1}$

$\rightarrow\left\langle V P^{4} V P V P^{2} V\right\rangle\left(\Delta E_{1}\right)^{4}$

241

$S_{2} S_{4} S_{1}=5 \rightarrow\left\langle V P^{2} V P^{2} V P V\right\rangle \Delta E_{1} \Delta E_{3}$

$\rightarrow-\left\langle V P^{2} V P^{3} V P V\right\rangle \Delta E_{1} \Delta E_{2} \Delta E_{1}$

$\rightarrow-\left\langle V P^{2} V P^{3} V P V\right\rangle\left(\Delta E_{1}\right)^{2} \Delta E_{2}$

$\rightarrow\left\langle V P^{2} V P^{4} V P V\right\rangle\left(\Delta E_{1}\right)^{4}$

214

$S_{2} S_{1} S_{4}=5 \rightarrow\left\langle V P^{2} V P V P^{2} V\right\rangle \Delta E_{1} \Delta E_{3}$

$\rightarrow-\left\langle V P^{2} V P V P^{3} V\right\rangle\left(\Delta E_{1}\right)^{2} \Delta E_{2}$

$\rightarrow-\left\langle V P^{2} V P V P^{3} V\right\rangle \Delta E_{1} \Delta E_{2} \Delta E_{1}$

$\rightarrow\left\langle V P^{2} V P V P^{4} V\right\rangle\left(\Delta E_{1}\right)^{4}$ 


\section{Continued}

$\rightarrow-\left\langle V P V P^{2} V P^{3} V\right\rangle\left(\Delta E_{1}\right)^{2} \Delta E_{2}$

$\rightarrow-\left\langle V P V P^{2} V P^{3} V\right\rangle \Delta E_{1} \Delta E_{2} \Delta E_{1}$

$\rightarrow\left\langle V P V P^{2} V P^{4} V\right\rangle\left(\Delta E_{1}\right)^{4}$

142

$S_{1} S_{5} S_{2}=5 \rightarrow\left\langle V P V P^{2} V P^{2} V\right\rangle \Delta E_{3} \Delta E_{1}$

$\rightarrow-\left\langle V P V P^{3} V P^{2} V\right\rangle \Delta E_{2}\left(\Delta E_{1}\right)^{2}$

$\rightarrow-\left\langle V P V P^{3} V P^{2} V\right\rangle \Delta E_{1} \Delta E_{2} \Delta E_{1}$

$\rightarrow\left\langle V P V P^{4} V P^{2} V\right\rangle\left(\Delta E_{1}\right)^{4}$

43

$S_{4} S_{3}=5 \times 2=10 \rightarrow\left\langle V P^{2} V P^{2} V\right\rangle \Delta E_{3} \Delta E_{2}$

$\rightarrow-\left\langle V P^{3} V P^{2} V\right\rangle \Delta E_{1}\left(\Delta E_{2}\right)^{2}$

$\rightarrow-\left\langle V P^{3} V P^{2} V\right\rangle \Delta E_{2} \Delta E_{1} \Delta E_{2}$

$\rightarrow\left\langle V P^{4} V P^{2} V\right\rangle\left(\Delta E_{1}\right)^{3} \Delta E_{2}$

$\rightarrow-\left\langle V P^{2} V P^{3} V\right\rangle \Delta E_{3}\left(\Delta E_{1}\right)^{2}$

$\rightarrow\left\langle V P^{3} V P^{3} V\right\rangle \Delta E_{2} \Delta E_{1}\left(\Delta E_{1}\right)^{2}$

$\rightarrow\left\langle V P^{3} V P^{3} V\right\rangle \Delta E_{1} \Delta E_{2}\left(\Delta E_{1}\right)^{2}$

$\rightarrow-\left\langle V P^{4} V P^{3} V\right\rangle\left(\Delta E_{1}\right)^{5}$

34

$S_{3} S_{4}=2 \times 5=10 \rightarrow\left\langle V P^{2} V P^{2} V\right\rangle \Delta E_{2} \Delta E_{3}$

$\rightarrow-\left\langle V P^{2} V P^{3} V\right\rangle \Delta E_{2} \Delta E_{1} \Delta E_{2}$

$\rightarrow-\left\langle V P^{2} V P^{3} V\right\rangle \Delta E_{2} \Delta E_{2} \Delta E_{1}$

$\rightarrow\left\langle V P^{2} V P^{4} V\right\rangle \Delta E_{2}\left(\Delta E_{1}\right)^{3}$

$\rightarrow-\left\langle V P^{3} V P^{2} V\right\rangle\left(\Delta E_{1}\right)^{2} \Delta E_{3}$

$\rightarrow\left\langle V P^{3} V P^{3} V\right\rangle\left(\Delta E_{1}\right)^{2} \Delta E_{1} \Delta E_{2}$

$\rightarrow\left\langle V P^{3} V P^{3} V\right\rangle\left(\Delta E_{1}\right)^{2} \Delta E_{2} \Delta E_{1}$

$\rightarrow-\left\langle V P^{3} V P^{4} V\right\rangle\left(\Delta E_{1}\right)^{5}$

52

$S_{5} S_{2}=14 \rightarrow\left\langle V P^{2} V P^{2} V\right\rangle \Delta E_{4} \Delta E_{1}$

$\rightarrow-\left\langle V P^{3} V P^{2} V\right\rangle \Delta E_{3}\left(\Delta E_{1}\right)^{2}$

$\rightarrow-\left\langle V P^{3} V P^{2} V\right\rangle\left(\Delta E_{2}\right)^{2} \Delta E_{1}$

$\rightarrow-\left\langle V P^{3} V P^{2} V\right\rangle \Delta E_{3}\left(\Delta E_{1}\right)^{2}$

$\rightarrow\left\langle V P^{4} V P^{2} V\right\rangle \Delta E_{2}\left(\Delta E_{1}\right)^{3}$

$\rightarrow\left\langle V P^{4} V P^{2} V\right\rangle \Delta E_{1} \Delta E_{2}\left(\Delta E_{1}\right)^{2}$

$\rightarrow\left\langle V P^{4} V P^{2} V\right\rangle\left(\Delta E_{1}\right)^{2} \Delta E_{2} \Delta E_{1}$ 


\section{Continued}

\begin{tabular}{|c|c|c|}
\hline & $\rightarrow-\left\langle V P^{5} V P^{2} V\right\rangle\left(\Delta E_{1}\right)^{5}$ & (1) \\
\hline \multirow[t]{8}{*}{25} & $S_{2} S_{5}=14 \rightarrow\left\langle V P^{2} V P^{2} V\right\rangle \Delta E_{1} \Delta E_{4}$ & (5) \\
\hline & $\rightarrow-\left\langle V P^{2} V P^{3} V\right\rangle\left(\Delta E_{1}\right)^{2} \Delta E_{3}$ & (2) \\
\hline & $\rightarrow-\left\langle V P^{2} V P^{3} V\right\rangle \Delta E_{1}\left(\Delta E_{2}\right)^{3}$ & (1) \\
\hline & $\rightarrow-\left\langle V P^{2} V P^{3} V\right\rangle \Delta E_{1} \Delta E_{3} \Delta E_{1}$ & (2) \\
\hline & $\rightarrow\left\langle V P^{2} V P^{4} V\right\rangle\left(\Delta E_{1}\right)^{3} \Delta E_{2}$ & (1) \\
\hline & $\rightarrow\left\langle V P^{2} V P^{4} V\right\rangle\left(\Delta E_{1}\right)^{2} \Delta E_{2} \Delta E_{1}$ & (1) \\
\hline & $\rightarrow\left\langle V P^{2} V P^{4} V\right\rangle \Delta E_{1} \Delta E_{2}\left(\Delta E_{1}\right)^{2}$ & (1) \\
\hline & $\rightarrow-\left\langle V P^{2} V P^{5} V\right\rangle\left(\Delta E_{1}\right)^{5}$ & (1) \\
\hline \multirow[t]{8}{*}{511} & $S_{5} S_{1}^{2}=14 \rightarrow-\left\langle V P^{2} V P V P V\right\rangle \Delta E_{4}$ & (5) \\
\hline & $\rightarrow\left\langle V P^{3} V P V P V\right\rangle \Delta E_{1} \Delta E_{3}$ & (2) \\
\hline & $\rightarrow\left\langle V P^{3} V P V P V\right\rangle\left(\Delta E_{2}\right)^{2}$ & (1) \\
\hline & $\rightarrow\left\langle V P^{3} V P V P V\right\rangle \Delta E_{3} \Delta E_{1}$ & (2) \\
\hline & $\rightarrow-\left\langle V P^{4} V P V P V\right\rangle\left(\Delta E_{1}\right)^{2} \Delta E_{2}$ & (1) \\
\hline & $\rightarrow-\left\langle V P^{4} V P V P V\right\rangle \Delta E_{1} \Delta E_{2} \Delta E_{1}$ & (1) \\
\hline & $\rightarrow-\left\langle V P^{4} V P V P V\right\rangle \Delta E_{2}\left(\Delta E_{1}\right)^{2}$ & (1) \\
\hline & $\rightarrow\left\langle V P^{5} V P V P V\right\rangle\left(\Delta E_{1}\right)^{4}$ & (1) \\
\hline \multirow[t]{8}{*}{151} & $S_{1}^{2} S_{5}=14 \rightarrow-\left\langle V P V P^{2} V P V\right\rangle \Delta E_{4}$ & (5) \\
\hline & $\rightarrow\left\langle V P V P^{3} V P V\right\rangle \Delta E_{1} \Delta E_{3}$ & (2) \\
\hline & $\rightarrow\left\langle V P V P^{3} V P V\right\rangle\left(\Delta E_{2}\right)^{2}$ & (1) \\
\hline & $\rightarrow\left\langle V P V P^{3} V P V\right\rangle \Delta E_{3} \Delta E_{1}$ & (2) \\
\hline & $\rightarrow-\left\langle V P V P^{4} V P V\right\rangle\left(\Delta E_{1}\right)^{2} \Delta E_{2}$ & (1) \\
\hline & $\rightarrow-\left\langle V P V P^{4} V P V\right\rangle \Delta E_{1} \Delta E_{2} \Delta E_{1}$ & (1) \\
\hline & $\rightarrow-\left\langle V P V P^{4} V P V\right\rangle \Delta E_{2}\left(\Delta E_{1}\right)^{2}$ & (1) \\
\hline & $\rightarrow\left\langle V P V P^{5} V P V\right\rangle\left(\Delta E_{1}\right)^{4}$ & (1) \\
\hline \multirow[t]{5}{*}{115} & $S_{1}^{2} S_{5}=14 \rightarrow-\left\langle V P V P V P^{2} V\right\rangle \Delta E_{4}$ & (5) \\
\hline & $\rightarrow\left\langle V P V P V P^{3} V\right\rangle \Delta E_{1} \Delta E_{3}$ & (2) \\
\hline & $\rightarrow\left\langle V P V P V P^{3} V\right\rangle\left(\Delta E_{2}\right)^{2}$ & (1) \\
\hline & $\rightarrow\left\langle V P V P V P^{3} V\right\rangle \Delta E_{3} \Delta E_{1}$ & (2) \\
\hline & $\rightarrow-\left\langle V P V P V P^{4} V\right\rangle\left(\Delta E_{1}\right)^{2} \Delta E_{2}$ & (1) \\
\hline
\end{tabular}




\section{Continued}

$\rightarrow-\left\langle V P V P V P^{4} V\right\rangle \Delta E_{1} \Delta E_{2} \Delta E_{1}$

$\rightarrow-\left\langle V P V P V P^{4} V\right\rangle \Delta E_{2}\left(\Delta E_{1}\right)^{2}$

$\rightarrow\left\langle V P V P V P^{5} V\right\rangle\left(\Delta E_{1}\right)^{4}$

61

$S_{6} S_{1}=42 \rightarrow-\left\langle V P^{2} V P V\right\rangle \Delta E_{5}$

$\rightarrow\left\langle V P^{3} V P V\right\rangle \Delta E_{4} \Delta E_{1}$

$\rightarrow\left\langle V P^{3} V P V\right\rangle \Delta E_{3} \Delta E_{2}$

$\rightarrow\left\langle V P^{3} V P V\right\rangle \Delta E_{2} \Delta E_{3}$

$\rightarrow\left\langle V P^{3} V P V\right\rangle \Delta E_{1} \Delta E_{4}$

$\rightarrow-\left\langle V P^{4} V P V\right\rangle \Delta E_{3}\left(\Delta E_{1}\right)^{2}$

$\rightarrow-\left\langle V P^{4} V P V\right\rangle\left(\Delta E_{2}\right)^{2} \Delta E_{1}$

$\rightarrow-\left\langle V P^{4} V P V\right\rangle\left(\Delta E_{1}\right)^{2} \Delta E_{3}$

$\rightarrow-\left\langle V P^{4} V P V\right\rangle \Delta E_{1}\left(\Delta E_{2}\right)^{2}$

$\rightarrow-\left\langle V P^{4} V P V\right\rangle \Delta E_{1}\left(\Delta E_{2}\right)^{2}$

$\rightarrow-\left\langle V P^{4} V P V\right\rangle\left(\Delta E_{1}\right)^{2} \Delta E_{3}$

$\rightarrow\left\langle V P^{5} V P V\right\rangle \Delta E_{2}\left(\Delta E_{1}\right)^{3}$

$\rightarrow\left\langle V P^{5} V P V\right\rangle \Delta E_{2}\left(\Delta E_{1}\right)^{3}$

$\rightarrow\left\langle V P^{5} V P V\right\rangle\left(\Delta E_{1}\right)^{3} \Delta E_{2}$

$\rightarrow\left\langle V P^{5} V P V\right\rangle\left(\Delta E_{1}\right)^{3} \Delta E_{2}$

$\rightarrow-\left\langle V P^{6} V P V\right\rangle\left(\Delta E_{1}\right)^{5}$

16

$S_{1} S_{6}=42 \rightarrow-\left\langle V P V P^{2} V\right\rangle \Delta E_{5}$

$\rightarrow\left\langle V P V P^{3} V\right\rangle \Delta E_{1} \Delta E_{4}$

$\rightarrow\left\langle V P V P^{3} V\right\rangle \Delta E_{2} \Delta E_{3}$

$\rightarrow\left\langle V P V P^{3} V\right\rangle \Delta E_{3} \Delta E_{2}$

$\rightarrow\left\langle V P V P^{3} V\right\rangle \Delta E_{4} \Delta E_{1}$

$\rightarrow-\left\langle V P V P^{4} V\right\rangle\left(\Delta E_{1}\right)^{2} \Delta E_{3}$

$\rightarrow-\left\langle V P V P^{4} V\right\rangle \Delta E_{1}\left(\Delta E_{2}\right)^{2}$

$\rightarrow-\left\langle V P V P^{4} V\right\rangle\left(\Delta E_{1}\right)^{2} \Delta E_{3}$

$\rightarrow-\left\langle V P V P^{4} V\right\rangle\left(\Delta E_{2}\right)^{2} \Delta E_{1}$

$\rightarrow-\left\langle V P V P^{4} V\right\rangle\left(\Delta E_{2}\right)^{2} \Delta E_{1}$

$\rightarrow-\left\langle V P V P^{4} V\right\rangle \Delta E_{3}\left(\Delta E_{1}\right)^{2}$ 


\section{Continued}

$$
\begin{aligned}
& \rightarrow\left\langle V P V P^{5} V\right\rangle\left(\Delta E_{1}\right)^{3} \Delta E_{2} \\
& \rightarrow\left\langle V P V P^{5} V\right\rangle\left(\Delta E_{1}\right)^{3} \Delta E_{2} \\
& \rightarrow\left\langle V P V P^{5} V\right\rangle \Delta E_{2}\left(\Delta E_{1}\right)^{3} \\
& \rightarrow\left\langle V P V P^{5} V\right\rangle \Delta E_{2}\left(\Delta E_{1}\right)^{3} \\
& \rightarrow-\left\langle V P V P^{6} V\right\rangle\left(\Delta E_{1}\right)^{5}
\end{aligned}
$$

\begin{tabular}{|c|c|c|}
\hline $1: 7$ & $\rightarrow-\left\langle V P^{2} V\right\rangle \Delta E_{6}$ & $\rightarrow S_{6}=(42)$ \\
\hline $1: 2: 7$ & $\rightarrow\left\langle V P^{3} V\right\rangle \Delta E_{1} \Delta E_{5}$ & $\rightarrow S_{1} S_{5}=(14)$ \\
\hline $1: 3: 7$ & $\rightarrow\left\langle V P^{3} V\right\rangle \Delta E_{2} \Delta E_{4}$ & $\rightarrow S_{2} S_{4}=(5)$ \\
\hline $1: 4: 7$ & $\rightarrow\left\langle V P^{3} V\right\rangle \Delta E_{3} \Delta E_{3}$ & $\rightarrow S_{3} S_{3}=(4)$ \\
\hline $1: 5: 7$ & $\rightarrow\left\langle V P^{3} V\right\rangle \Delta E_{4} \Delta E_{2}$ & $\rightarrow S_{4} S_{2}=(5)$ \\
\hline $1: 6: 7$ & $\rightarrow\left\langle V P^{3} V\right\rangle \Delta E_{5} \Delta E_{1}$ & $\rightarrow S_{5} S_{1}=(14)$ \\
\hline $1: 2: 3: 7$ & $\rightarrow-\left\langle V P^{4} V\right\rangle\left(\Delta E_{1}\right)^{2} \Delta E_{4}$ & $\rightarrow S_{1}^{2} S_{4}=(5)$ \\
\hline $1: 2: 4: 7$ & $\rightarrow-\left\langle V P^{4} V\right\rangle \Delta E_{1} \Delta E_{2} \Delta E_{3}$ & $\rightarrow S_{1} S_{2} S_{3}=(2)$ \\
\hline $1: 2: 5: 7$ & $\rightarrow-\left\langle V P^{4} V\right\rangle \Delta E_{1} \Delta E_{3} \Delta E_{2}$ & $\rightarrow S_{1} S_{3} S_{2}=(2)$ \\
\hline $1: 2: 6: 7$ & $\rightarrow-\left\langle V P^{4} V\right\rangle \Delta E_{1} \Delta E_{4} \Delta E_{1}$ & $\rightarrow S_{1} S_{4} S_{1}=(5)$ \\
\hline $1: 3: 4: 7$ & $\rightarrow-\left\langle V P^{4} V\right\rangle \Delta E_{1} \Delta E_{2} \Delta E_{3}$ & $\rightarrow S_{1} S_{2} S_{3}=(2)$ \\
\hline $1: 3: 5: 7$ & $\rightarrow-\left\langle V P^{4} V\right\rangle \Delta E_{2} \Delta E_{2} \Delta E_{2}$ & $\rightarrow S_{2}^{3}=(1)$ \\
\hline $1: 3: 6: 7$ & $\rightarrow-\left\langle V P^{4} V\right\rangle \Delta E_{2} \Delta E_{3} \Delta E_{1}$ & $\rightarrow S_{2} S_{3} S_{1}=(2)$ \\
\hline $1: 4: 5: 7$ & $\rightarrow-\left\langle V P^{4} V\right\rangle \Delta E_{3} \Delta E_{1} \Delta E_{2}$ & $\rightarrow S_{3} S_{1} S_{2}=(2)$ \\
\hline $1: 4: 6: 7$ & $\rightarrow-\left\langle V P^{4} V\right\rangle \Delta E_{3} \Delta E_{2} \Delta E_{1}$ & $\rightarrow S_{3} S_{2} S_{1}=(2)$ \\
\hline $1: 5: 6: 7$ & $\rightarrow-\left\langle V P^{4} V\right\rangle \Delta E_{4}\left(\Delta E_{1}\right)^{2}$ & $\rightarrow S_{4} S_{1}^{2}=(5)$ \\
\hline $1: 2: 3: 4: 7$ & $\rightarrow\left\langle V P^{5} V\right\rangle\left(\Delta E_{1}\right)^{3} \Delta E_{3}$ & $\rightarrow S_{1}^{3} S_{3}=(2)$ \\
\hline $1: 2: 3: 5: 7$ & $\rightarrow\left\langle V P^{5} V\right\rangle\left(\Delta E_{1}\right)^{2}\left(\Delta E_{2}\right)^{2}$ & $\rightarrow S_{1}^{2} S_{2}^{2}=(1)$ \\
\hline $1: 2: 3: 6: 7$ & $\rightarrow\left\langle V P^{s} V\right\rangle\left(\Delta E_{1}\right)^{2} \Delta E_{2} \Delta E_{1}$ & $\rightarrow S_{1}^{3} S_{3}=(2)$ \\
\hline $1: 2: 4: 5: 7$ & $\rightarrow\left\langle V P^{5} V\right\rangle \Delta E_{1} \Delta E_{2} \Delta E_{1} \Delta E_{2}$ & $\rightarrow S_{1}^{2} S_{2}^{2}=(1)$ \\
\hline $1: 2: 4: 6: 7$ & $\rightarrow\left\langle V P^{s} V\right\rangle \Delta E_{1}\left(\Delta E_{2}\right)^{2} \Delta E_{1}$ & $\rightarrow S_{1}^{2} S_{2}^{2}=(1)$ \\
\hline
\end{tabular}

Table 6. $N=8$. Perturbation terms due to the highest partition number of $N-1=7$. Total number of the perturbation terms obtained in the present Table:

$(42)+3 \times(14)+(5)+(14)+(9)+(5)+(5)+(4)+(6)=(132)=S_{7}$. Total number of the perturbation terms in Tables 4-6: $(93)+(204)+132=(429)=S_{8}$. 


\section{Continued}

$\begin{array}{lll}1: 2: 5: 6: 7 & \rightarrow\left\langle V P^{5} V\right\rangle \Delta E_{1} \Delta E_{3}\left(\Delta E_{1}\right)^{2} & \rightarrow S_{1} S_{3} S_{1}^{2}=(2) \\ 1: 3: 4: 5: 7 & \rightarrow\left\langle V P^{5} V\right\rangle \Delta E_{2}\left(\Delta E_{1}\right)^{2} \Delta E_{2} & \rightarrow S_{2} S_{1}^{3} S_{2}=(1) \\ 1: 3: 4: 6: 7 & \rightarrow\left\langle V P^{5} V\right\rangle \Delta E_{2} \Delta E_{1} \Delta E_{2} \Delta E_{1} & \rightarrow S_{2} S_{1} S_{2} S_{1}=(1) \\ 1: 3: 5: 6: 7 & \rightarrow\left\langle V P^{5} V\right\rangle\left(\Delta E_{2}\right)^{2}\left(\Delta E_{1}\right)^{2} & \rightarrow S_{2}^{2} S_{1}^{2}=(1) \\ 1: 4: 5: 6: 7 & \rightarrow\left\langle V P^{5} V\right\rangle \Delta E_{3}\left(\Delta E_{1}\right)^{3} & \rightarrow S_{3} S_{1}^{3}=(2) \\ 1: 2: 3: 4: 5: 7 & \rightarrow-\left\langle V P^{6} V\right\rangle\left(\Delta E_{1}\right)^{4} \Delta E_{2} & \rightarrow S_{1}^{4} S_{2}=(1) \\ 1: 2: 3: 4: 6: 7 & \rightarrow-\left\langle V P^{6} V\right\rangle\left(\Delta E_{1}\right)^{3} \Delta E_{2} \Delta E_{1} & \rightarrow S_{1}^{3} S_{2} S_{1}=(1) \\ 1: 2: 3: 5: 6: 7 & \rightarrow-\left\langle V P^{6} V\right\rangle\left(\Delta E_{1}\right)^{2} \Delta E_{2}\left(\Delta E_{1}\right)^{2} & \rightarrow S_{1}^{2} S_{2} S_{1}^{2}=(1) \\ 1: 2: 4: 5: 6: 7 & \rightarrow-\left\langle V P^{6} V\right\rangle \Delta E_{1} \Delta E_{2}\left(\Delta E_{1}\right)^{3} & \rightarrow S_{1} S_{2} S_{1}^{3}=(1) \\ 1: 3: 4: 5: 6: 7 & \rightarrow-\left\langle V P^{6} V\right\rangle \Delta E_{2}\left(\Delta E_{1}\right)^{4} & \rightarrow S_{2} S_{1}^{4}=(1) \\ 1: 2: 3: 4: 5: 6: 7 & \rightarrow\left\langle V P^{7} V\right\rangle\left(\Delta E_{1}\right)^{6} & \rightarrow S_{1}^{6}=(1)\end{array}$

actual paper-for an example. To this purpose we choose the calculation of $N=6$ being the most developed case considered in Appendix of [34]. The mentioned data of Appendix are next compared with the corresponding data due to the present method; see Table 7.

On the left-hand side of Table 7 are presented the symbols of the perturbation terms applied in the partition notation of the present paper, on the right-hand side of Table 7 the method represented in Appendix of [34] is applied.

There exists a full agreement of the data obtained in the present paper with those taken from Appendix of [34].

\section{Summary: General Properties of the Scale of Time Suitable to Calculate the Schrödinger Perturbation Energy}

One of the fundamental processes of quantum mechanics is a change of a given system upon the action of some perturbation potential which-in its character-can be independent of time, but is dependent solely on the particle coordinates. To calculate the result of such a change acting on a non-degenerate quantum system, the Schrödinger perturbation formalism-represented by the sets of energy terms labelled by orders $N$-is required. In principle no time approach, or time parameter, should be used to this purpose.

We are guided, however, by the Leibniz idea that a suitable arrangement of the physical events along a time scale can be helpful in an analysis of any system change, including the perturbation effect. Consequently, by assuming that a change of a system-also due to the action of a time-independent perturbation potential-requires some interval of time, a sequence and origin of the time moments entering such interval can be of importance.

In principle there exist many ways according to which the necessary sets of 
Table 7. Comparison of the energy terms calculated in Appendix of [34] with those obtained in the present paper: an example giving the terms belonging to the order $N=6$. The first 14 terms presented in the right-hand side column are calculated-according to [34] -automatically on the basis of the results obtained for $N=5$.

\begin{tabular}{|c|c|c|}
\hline Number of the term & $\begin{array}{l}\text { Partition symbol applied } \\
\text { in the present method }\end{array}$ & Energy result obtained in [34] \\
\hline 1 & 11111 & $\langle V P V P V P V P V P V\rangle$ \\
\hline 2 & 2111 & $-\left\langle V P^{2} V P V P V P V\right\rangle \Delta E_{1}$ \\
\hline 3 & $311\left(1^{\text {st }}\right.$ term $)$ & $-\left\langle V P^{2} V P V P V\right\rangle \Delta E_{2}$ \\
\hline 4 & $311\left(2^{\text {nd }}\right.$ term $)$ & $\left\langle V P^{3} V P V P V\right\rangle\left(\Delta E_{1}\right)^{2}$ \\
\hline 5 & 1211 & $-\left\langle V P V P^{2} V P V P V\right\rangle \Delta E_{1}$ \\
\hline 6,7 & $41\left(1^{\text {st }}\right.$ term $)$ & $-\left\langle V P^{2} V P V\right\rangle \Delta E_{3}$ \\
\hline 8 & $41\left(2^{\text {nd }}\right.$ term $)$ & $\left\langle V P^{3} V P V\right\rangle \Delta E_{1} \Delta E_{2}$ \\
\hline 9 & $41\left(3^{\text {rd }}\right.$ term $)$ & $\left\langle V P^{3} V P V\right\rangle \Delta E_{2} \Delta E_{1}$ \\
\hline 10 & $41\left(4^{\text {th }}\right.$ term $)$ & $-\left\langle V P^{4} V P V\right\rangle\left(\Delta E_{1}\right)^{3}$ \\
\hline 11 & $131\left(1^{\mathrm{st}}\right.$ term $)$ & $-\left\langle V P V P^{2} V P V\right\rangle \Delta E_{2}$ \\
\hline 12 & $131\left(2^{\text {nd }}\right.$ term $)$ & $\left\langle V P V P^{3} V P V\right\rangle\left(\Delta E_{1}\right)^{2}$ \\
\hline 13 & 1121 & $-\left\langle V P V P V P^{2} V P V\right\rangle \Delta E_{1}$ \\
\hline 14 & 221 & $\left\langle V P^{2} V P^{2} V P V\right\rangle \Delta E_{1}$ \\
\hline $15-19$ & $5\left(1^{\text {st }}\right.$ term $)$ & $-\left\langle V P^{2} V\right\rangle \Delta E_{4}$ \\
\hline 20,21 & $5\left(2^{\text {nd }}\right.$ term $)$ & $\left\langle V P^{3} V\right\rangle \Delta E_{1} \Delta E_{3}$ \\
\hline 22 & $5\left(3^{\text {rd }}\right.$ term $)$ & $\left\langle V P^{3} V\right\rangle\left(\Delta E_{2}\right)^{2}$ \\
\hline 23,24 & $5\left(4^{\text {th }}\right.$ term $)$ & $\left\langle V P^{3} V\right\rangle \Delta E_{3} \Delta E_{1}$ \\
\hline 25 & $5\left(5^{\text {th }}\right.$ term $)$ & $-\left\langle V P^{4} V\right\rangle\left(\Delta E_{1}\right)^{2} \Delta E_{2}$ \\
\hline 26 & $5\left(6^{\text {th }}\right.$ term $)$ & $-\left\langle V P^{4} V\right\rangle \Delta E_{1} \Delta E_{2} \Delta E_{1}$ \\
\hline 27 & $5\left(7^{\text {th }}\right.$ term $)$ & $-\left\langle V P^{4} V\right\rangle \Delta E_{2}\left(\Delta E_{1}\right)^{2}$ \\
\hline 28 & $5\left(8^{\text {th }}\right.$ term $)$ & $\left\langle V P^{s} V\right\rangle\left(\Delta E_{1}\right)^{4}$ \\
\hline 29,30 & $14\left(1^{\text {st }}\right.$ term $)$ & $-\left\langle V P V P^{2} V\right\rangle \Delta E_{3}$ \\
\hline 31 & $14\left(2^{\text {nd }}\right.$ term $)$ & $\left\langle V P V P^{3} V\right\rangle \Delta E_{1} \Delta E_{2}$ \\
\hline 32 & $14\left(3^{\text {rd }}\right.$ term $)$ & $\left\langle V P V P^{3} V\right\rangle \Delta E_{2} \Delta E_{1}$ \\
\hline 33 & $14\left(4^{\text {th }}\right.$ term $)$ & $\left\langle V P V P^{4} V\right\rangle\left(\Delta E_{1}\right)^{4}$ \\
\hline 34 & $113\left(1^{\text {st }}\right.$ term $)$ & $-\left\langle V P V P V P^{2} V\right\rangle \Delta E_{2}$ \\
\hline 35 & $113\left(2^{\text {nd }}\right.$ term $)$ & $\left\langle V P V P V P^{3} V\right\rangle\left(\Delta E_{1}\right)^{2}$ \\
\hline
\end{tabular}




\section{Continued}

\begin{tabular}{ccc}
\hline 36 & $23\left(1^{\text {st }}\right.$ term $)$ & $\left\langle V P^{2} V P^{2} V\right\rangle \Delta E_{1} \Delta E_{2}$ \\
37 & $23\left(2^{\text {nd }}\right.$ term $)$ & $-\left\langle V P^{2} V P^{3} V\right\rangle\left(\Delta E_{1}\right)^{3}$ \\
38 & 1112 & $-\left\langle V P V P V P V P^{3} V\right\rangle \Delta E_{1}$ \\
39 & 212 & $\left\langle V P^{2} V P V P^{2} V\right\rangle\left(\Delta E_{1}\right)^{2}$ \\
40 & $32\left(1^{\text {st }}\right.$ term $)$ & $\left\langle V P^{2} V P^{2} V\right\rangle \Delta E_{2} \Delta E_{1}$ \\
41 & $32\left(2^{\text {nd }}\right.$ term $)$ & $-\left\langle V P^{3} V P^{2} V\right\rangle\left(\Delta E_{1}\right)^{3}$ \\
42 & 122 & $\left\langle V P V P^{2} V P^{2} V\right\rangle\left(\Delta E_{1}\right)^{2}$
\end{tabular}

terms representing the perturbation effect can be calculated. But we find that a circular scale of time-accompanied by the time-point contractions on it-can represent in full the necessary terms belonging to a particular order of the perturbation energy. These terms become obtainable on the basis of the time scale in an almost automatic way. Only the change of the perturbation order-associated with a change of the number of time points considered on the scale-implies a progressive action attributed to time.

A full presentation of the perturbation terms has been done for orders $N=7$ and $N=8$; the terms of the lower $N$ are accessible in the literature presented before [25]-[34].

At the first sight it seems that the paper has only a purely mathematical background. In fact the aim is to solve a definite Schrödinger differential equation, but the way to do that is to solve first a presumably more simple equation. Next the solutions of that more simple equation should be combined into those belonging to a more complicated problem.

Both equations are assumed to be different by a potential change independent of time. In fact the time parameter neither enters the actual perturbation equation, nor the equation representing a former more simple problem. Nevertheless the change of the potential-equivalent to the change of the Hamiltonian operator between the unperturbed and perturbed equations-occupies some time. We assume the time of the potential change as negligibly small. A much more longer time, therefore of a non-negligible size, is expected to be occupied as an effect of the original potential change. This is so because the perturbed, i.e. originally unstable system, should wait to occupy one of its stationary states. In effect the time, required to make the perturbed system equivalent to a stationary object, can be long. An estimate of the size of that interval is beyond of our ability. Mathematically however, the both states, unperturbed and perturbed one, are both accessible and can be defined without any reference to the notion of time. So a question may arise what is the role of time-if any such role does exist-in the perturbation theory?

With the absence of any time intervals in the formalism, the answer is that time is an ordering parameter. Moreover this role is rather of a gradual character 
because it does not concern the perturbation process as a whole, but is decisive in the successive steps of that process. In fact the perturbation effect can be separated into parts called the perturbation orders. Any order $N$ is characterized by: 1) a definite number of the Huby-Tong kinds of the perturbation terms specified by the formula $(21) ; 2)$ a constant number of $N-1$ terms $P$ and number of $N$ terms $V$ entering any perturbation term belonging to the order $N$.

But beyond of the number of terms characteristic for a given $N$, an important role plays the sequence of the "collision" events of an unperturbed system with the perturbation potential. This sequence follows a closed scale of time equivalent to a topological circle. Any scale representing some $N$ th perturbation order has $N$ time points on it. Among these points only one-called the beginning-end point-is free from contractions. Other time points on the scale, being $N-1$ in their number, can be submitted to contractions in a definite way. The contractions give the corresponding (closed) loops of time discussed in Sec. 4 and consequently the expected corrections to energy.

As an effect of contractions variable with $N$, the number of kinds of the perturbation terms increased by a single term due to an uncontracted loop of time characteristic for a given $N$, becomes equal to the number $S_{N}$ presented in the formula (21). The arrangement of the time points in the contractions is providing us with the side loops of time corresponding to contractions. After combining the energy contributions due to the side loops with those given by the main loop of time, we arrive at the proper Schrödinger perturbation terms for energy of a given order $N$. No supplementary calculations are required to attain that purpose.

In fact the shape of the time scale, together with the time-point contractions done on it, play a decisive role in calculating all kinds of the Schrödinger perturbation terms entering a given order $N$. This result is not proved in an exact way but obtained in course of the systematic energy calculations belonging to the individual $N$ s.

\section{A Philosophical Background Concerning the Present Results}

A philosophical background of the results obtained in the paper seems to be twofold. The first aim was to obtain a general look on the shape of the time scale. A principal point becomes here to get a real relevance of the question of the direction of time, or more simply the problem of sequence of the time events, to some physical process [35]. Let us note here an opinion that the theory of the whole world time is a redundant concept-one only needs a knowledge of world's possible configurations [36].

A reply in the present case is that if the scales belonging to individual $N$ are considered, their shape is evidently a closed line. A characteristic circular-like character of the time scale suitable to calculations of the perturbation energy for a given $N$ seems to be not a unique property in physics; see e.g. Rey [37] and Zawirski quoted in [38]. A much discussed reference which can be cited here is 
the paper by Gödel [39]. In an analysis of cosmological solutions concerning the Einstein's field equations for gravitation he remarked that: (a) it is not possible to assign a time coordinate $t$ to each space-time point in such a way that $t$ always increases, if one moves in a positive time-like direction, and this holds for both for an open and a closed time coordinate; (b) every world line of matter occurring in the solution is an open line of an infinite length which approaches any of its preceding points again; (c) there also exist closed time-like lines.

In fact a total scale of time applied in the present paper-because of an increasing number of collisions with the potential $V^{\text {per }}$-does increase gradually with $N$. This makes the second step of the time way, i.e. that due to an increase of $N$, similar to an infinite linear scale referred in (1).

\section{Conflicts of Interest}

The author declares no conflicts of interest regarding the publication of this paper.

\section{References}

[1] Schrödinger, E. (1926) Quantisierung als Eigenwertproblem (erste Mitteilung). Annalen der Physik, 79, 361-376.

[2] Schrödinger, E. (1926) Quantisierungals Eigenwert Problem (zweite Mitteilung). Annalen der Physik, 79, 489-527.

[3] Schrödinger, E. (1926) Quantisierung als Eigenwertproblem (dritte Mitteilung: Storungstheorie, mit Anwendung auf den Starkeffekt der Balmerlinien). Annalen der Physik, 80, 437-490.

[4] Schrödinger, E. (1926) Quantisierung als Eigenwertproblem (vierte Mitteilung). Annalen der Physik, 81, 109-139.

[5] Schiff, L.I. (1968) Quantum Mechanics. 3rd Edition, McGraw-Hill, New York.

[6] Huby, R. (1961) Formulae for Non-Degenerate Raleigh-Schrodinger Perturbation Theory. Proceedings of the Physical Society (London), 78, 529.

[7] Tong, B.Y. (1962) On Huby's Rules for Non-Degenerate Rayleigh-Schrodinger Perturbation Theory of Any Order. Proceedings of the Physical Society (London), 80, 1101 .

[8] Morse, P.M. and Feshbach, H. (1953) Methods of Theoretical Physics. Vol. 2, McGraw-Hill, New York.

[9] Bloch, C. (1958) Sur la théorie des perturbations des états liés Claude Bloch. Nuclear Physics, 6, 329-347.

[10] Brueckner, K.A. (1955) Many-Body Problem for Strongly Interacting Particles. II. Linked Cluster Expansion. Physical Review, 100, 36.

https://doi.org/10.1103/PhysRev.100.36

[11] Goldstone, J. (1957) Derivation of the Brueckner Many-Body Theory. Proceedings of the Royal Society of London. Series A, 329, 267.

[12] Löwdin, P.O. (1966) The Calculation of Upper and Lower Bounds of Energy Eigenvalues in the Perturbation Theory by Means of Partitioning Techniques. In: Wilcox, C.H., Ed., Peturbation Theory and Its Application in Quantum Mechanics, Wiley, New York, 255. 
[13] March, N.H., Young, W.H. and Sampanthar, S. (1967) The Many-Body Problem in Quantum Mechanics. Cambridge University Press, London.

[14] Paldus, J. and Cizek, J. (1975) Time-Independent Diagrammatic Approach to Perturbation Theory of Fermion Systems. Advances in Quantum Chemistry, 9, 105-197. https://doi.org/10.1016/S0065-3276(08)60040-4

[15] Raimes, S. (1972) Many-Electron Theory. North-Holland, Amsterdam.

[16] Salzman, W.R. (1968) Diagrammatical Derivation and Representation of Rayleigh-Schrödinger Perturbation Theory. Journal of Chemical Physics, 49, 3035. https://doi.org/10.1063/1.1670546

[17] Silverstone, H.J. and Holloway, T.T. (1970) Explicit Formulas for the Nth-Order Wavefunction and Energy in Nondegenerate Rayleigh-Schrödinger Perturbation Theory. Journal of Chemical Physics, 52, 1472. https://doi.org/10.1063/1.1673153

[18] Feynman, R.P. (1966) The Development of the Space-Time View of Quantum Electrodynamics. Science, 153, 699-708. https://doi.org/10.1126/science.153.3737.699

[19] Mattuck, R.D. (1976) A Guide to Feynman Diagrams in a Many-Body Problem. 2nd Edition, McGraw Hill, New York.

[20] Ziman, J.M. (1969) Elements of Advanced Quantum Theory. Cambridge University Press, Cambridge.

[21] Leibniz, G.W. (1924) Hauptschriften zur Grundlagen der Philosophie. Leipzig, Vol. $1,329$.

[22] Rescher, N. (2013) On Leibniz. University of Pittsburgh Press, Pittsburgh. https://doi.org/10.2307/j.ctt7zw8g2

[23] Leibniz, G.W. (1956) In: Alexander, H.H., Ed., The Leibniz-Clarke Correspondence (1716), Manchester University Press, Manchester, 69.

[24] Barbour, J.B. and Bertotti, B. (1982) Mach's Principle and the Structure of Dynamical Theories. Proceedings of the Royal Society A (London), 382, 295.

[25] Olszewski, S. (1991) Time Scale and Its Application in Perturbation Theory. Zeitschrift fuer Naturforschung A, 46, 313-320. https://doi.org/10.1515/zna-1991-0404

[26] Olszewski, S. and Kwiatkowski, T. (1998) A Topological Approach to Evaluation of Non-Degenerate Schrödinger Perturbation Energy Based on a Circular Scale of Time. Computers in Chemistry, 22, 445-461. https://doi.org/10.1016/S0097-8485(98)00023-0

[27] Olszewski, S. (2003) Two Pathways of the Time Parameter Characteristic for the Perturbation Problem in Quantum Chemistry. Trends in Physical Chemistry, 9, 69.

[28] Olszewski, S. (2013) A Look on the Scale of Time Useful in Non-Relativistic Quantum Mechanics. Quantum Matter, 2, 481-483. https://doi.org/10.1166/qm.2013.1085

[29] Olszewski, S. (2014) Circular Scale of Time and Energy of a Quantum State Calculated from the Schrodinger Perturbation Theory. Circular Scale of Time and Construction of the Schrodinger Perturbation Series for Energy Made Simple. Journal of Modern Physics, 5, 1502.

[30] Olszewski, S. (2014) Circular Scale of Time as a Way of Calculating the Quantum-Mechanical Perturbation Energy Given by the Schrödinger Method. Journal of Quantum Information Science, 4, 269. https://doi.org/10.4236/jqis.2014.44022

[31] Olszewski, S. (2015) Classical Mechanics, Quantum Mechanics and Time Development of the Schroedinger Perturbation Process. Quantum Matter, 4, 523-532. https://doi.org/10.1166/qm.2015.1227 
[32] Olszewski, S. (2004) Kolowaskalaczasu z punktu widzeniamechanikikwantowej w ujeciu Schrodingera. Studia Philosophiae Christianae, 40, 57. (In Polish)

[33] Olszewski, S. (2011) Circular Scale of Time Applied in Classifying the Quantum-Mechanical Energy Terms Entering the Framework of the Schrödinger Perturbation Theory. Journal of Quantum Information Science, 1, 142-148. https://doi.org/10.4236/jqis.2011.13020

[34] Olszewski, S. (2018) Circular Time Scale Yields a Recurrent Calculation of the Schrödinger Perturbation Energy. Journal of Modern Physics, 9, 1491-1521. https://doi.org/10.4236/jmp.2018.98093

[35] Sklar, L. (1974) Space, Time and Spacetime. University of California Press, Oakland.

[36] Barbour, J.B. (1994) The Emergence of Time and Its Arrow from Timelessness. In: Halliwell, J.J., Perez-Mercader, J. and Zurek, W.H., Eds., Physical Origins of Time Asymmetry, Cambridge University Press, Cambridge, 405.

[37] Rey, A. (1927) Le retour eternel et la philosophie de la physique. Paris. (In French)

[38] Szumilewicz, I. (1964) On the Direction of the Time-Flow. Państwowe Wydawnictwo Naukowe, Warszawa. (In Polish)

[39] Gödel, K. (1949) An Example of a New Type of Cosmological Solutions of Einstein's Field Equations of Gravitation. Reviews of Modern Physics, 21, 447.

https://doi.org/10.1103/RevModPhys.21.447 